\title{
Intermediation in Markets for Goods and Markets for Assets
}

\author{
Ed Nosal, Yuet-Yee Wong, and Randall Wright
}

\section{Working Paper 2019-5 \\ March 2019}

\begin{abstract}
We analyze agents' decisions to act as producers or intermediaries using equilibrium search theory. Extending previous analyses in various ways, we ask when intermediation emerges and study its efficiency. In one version of the framework, meant to resemble retail, middlemen hold goods, which entails (storage) costs; that model always displays uniqueness and simple transition dynamics. In another version, middlemen hold assets, which entails negative costs, that is, positive returns; that model can have multiple equilibria and complicated belief-based dynamics. These results are consistent with the venerable view that intermediation in financial markets is more prone to instability than in goods markets.
\end{abstract}

JEL classification: G24, D83

Key words: middlemen, intermediation, search, bargaining, multiplicity

https://doi.org/10.29338/wp2019-05

An earlier version of this paper circulated as "Who Wants To Be A Middleman?" For input, the authors thank Karl Shell, Alberto Trejos, Steve Williamson, Gregor Jarosch, Giorgia Piacentino, and seminar participants at the Chicago Fed, Minneapolis Fed, Philadelphia Fed, Cornell, Simon Fraser, UNC, Wisconsin, the Canadian Macro Study Group, and the St. Louis Fed/Tsinghua Monetary Policy Conference in Beijing. The authors especially thank Yu Zhu, who helped with the numerical work. Wright acknowledges support from the Ray Zemon Chair in Liquid Assets at the Wisconsin School of Business. The views expressed here are those of the authors and not necessarily those of the Federal Reserve Bank of Atlanta or the Federal Reserve System. Any remaining errors are the authors' responsibility.

Please address questions regarding content to Ed Nosal, Research Department, Federal Reserve Bank of Atlanta, 1000 Peachtree Street NE, Atlanta, GA 30309-4470, 404-498-8814, ed.nosal@atl.frb.org; Yuet-Yee Wong, Department of Economics, Binghamton University, P.0. Box 6000, Binghamton, NY 13902, yywong01@gmail.com; or Randall Wright, School of Business, University of Wisconsin-Madison and NBER, Grainger Hall, 975 University Avenue, Madison, WI 53706, rwright@bus.wisc.edu.

Federal Reserve Bank of Atlanta working papers, including revised versions, are available on the Atlanta Fed's website at www.frbatlanta.org. Click "Publications" and then "Working Papers." To receive e-mail notifications about new papers, use frbatlanta.org/forms/subscribe. 


\section{Introduction}

This paper studies intermediation in markets for goods and markets for assets, building on the search-and-bargaining framework of Rubinstein and Wolinsky (1987), hereafter RW. The analysis extends existing versions of RW on several dimensions, and in particular, we endogenize market composition by letting agents choose to act as either middlemen or producers. This leads to the following: a version of the model designed to resemble goods markets, in a simple but reasonable way, has a unique equilibrium under standard assumptions; a version designed to resemble asset markets, under similar assumptions, can have multiple equilibria that can be ranked in terms of efficiency. For this result the sets of producers and middlemen must be endogenous - if they are exogenous uniqueness obtains in markets for assets as well as goods.

As background, first note that the original RW environment had no cost of production or search, equal numbers of producers and consumers, symmetric bargaining, and a fixed number of middlemen. In equilibrium middlemen participate in the market iff they have a better search technology than producers, as is efficient. In Nosal et al. (2015) we extended this to allow more general bargaining and costs, but that was relatively straightforward because, following RW, we maintained linear utility, only considered steady states, restricted inventories to $\{0,1\}$, and kept the sets of producers and middlemen fixed. This paper relaxes all of these restrictions, with the key generalization being that agents decide to act as producers or middlemen, and that requires a rather different approach. ${ }^{1}$

\footnotetext{
${ }^{1}$ For experts in search theory we can explain why it requires a different approach, although this can be skipped without loss of continuity. First, previous RW models take the arrival rates $\boldsymbol{\alpha}=\left\{\alpha_{i j}\right\}$ as exogenous, where $\alpha_{i j}$ is the rate at which type $i$ meets type $j$. Under certain conditions that is legitimate because there exists a distribution of types, say $\mathbf{n}=\left\{n_{i}\right\}$, consistent with $\boldsymbol{\alpha}$, uniform random matching, and the identities implied by bilateral meetings, $n_{i} \alpha_{i j}=n_{j} \alpha_{j i}$. While it is convenient to take $\boldsymbol{\alpha}$ as fixed, we cannot do so when $\mathbf{n}$ is endogenous. Hence, we use uniform random matching, $\alpha_{i j}=\alpha_{i} n_{j}$, where $\alpha_{i}$ is a baseline arrival rate for type $i$. But now the relevant identities imply $\alpha_{i}=\alpha \forall i$, and in particular $\alpha_{p c}=\alpha_{m c}$, so we must abandon RW's idea that middlemen are useful when $\alpha_{m c}>\alpha_{p c}$. Fortunately, other factors here take over for arrival rates, including bargaining powers and storage costs or returns.
} 
We then distinguish between markets for goods and markets for assets as follows: as in retail markets, holding inventories of goods entails a storage cost; holding inventories of assets instead entails a positive return, or a negative cost, as is potentially the case with, e.g., houses, art, productive capital, etc. Now there can also be costs to safely storing assets, but it is worth considering the case where the net return is positive, because that is necessary (not sufficient) for generating multiple steady states and endogenous dynamics. Of course, it is well known that one can generate multiplicity and dynamics in search theory with a variety of other devices, e.g., increasing returns in matching or production technologies (Diamond 1982; Mortensen 1999). We eschew those devices to concentrate on something new, complementarities in decisions by middlemen to adopt either buy-and-hold or buy-and-sell strategies.

Our producers generate goods, or assets, like capital, and trade them to end users. They may also trade them to middlemen, who may or may not trade them to end users. With goods as defined here (storage has a cost) the trading decision of a middleman meeting an end user is trivial, because buy-and-hold strategies cannot be optimal with negative returns. With assets as defined here (storage has a positive return) the decision is not trivial. To motivate why this is interesting, our asset market intermediaries can be interpreted in a stylized way as financial institutions, acquiring capital from originators and choosing when to pass it on. Our results thus provide support for the notion that financial institutions are less stable than other intermediaries, since we get multiplicity and belief-based volatility in intermediated markets for assets but not goods. ${ }^{2}$

For the intuition, suppose first that middlemen pass capital inventories on to

\footnotetext{
${ }^{2}$ The venerable notion that financial intermediation engenders instability/fragility is commonly associated with names like Minsky, Kindleberger and Keynes; for more recent expositions see, e.g., Akerlof and Shiller (2009) or Reinhart and Rogoff (2009). As regards banking, in particular, Rolnick and Weber (1986) say "Historically, even some of the staunchest proponents of laissez-faire have viewed banking as inherently unstable and so requiring government intervention." One such proponent is Friedman (1960), who opposed regulation of virtually everything except banks. Now this paper is not about unstable banking or credit arrangements (see Vives 2016 and $\mathrm{Gu}$ et al. 2013 on those issues); it is about instability in intermediated asset markets, but that seems at least as relevant.
} 
end users. Then lots of middlemen will be searching for new capital, leading to high producer profit and hence many producers. With more producers in the market it is easier for middlemen to get capital, thus rationalizing their decision to trade it away and making active intermediation an equilibrium for some parameters. Now suppose middlemen keep capital for themselves. Then they are more likely to already have capital, leading to lower profits and fewer producers. This makes it harder for middlemen to get capital, thus rationalizing their decision to not trade it away in another equilibrium for the same parameters. Moreover, the equilibria can be welfare ranked - having middlemen trade with end users is better - and, again, the multiplicity can only arise when the return on inventories is positive and when the composition of the market is endogenous.

Section 2 describes a general benchmark environment. Then we analyze markets for goods and markets for assets separately, the former in Sections 3-4, and the latter in Sections 5-7. Section 8 discusses robustness, including, in particular, an extension that allows general inventories, while the baseline model restricts inventories to $\{0,1\} .{ }^{3}$ Section 9 contains concluding remarks. Some other extensions and technical proofs are in the Appendices. ${ }^{4}$

\footnotetext{
${ }^{3}$ Having inventories in $\{0,1\}$ is special, although as in search theory going back to Diamond (1982) it allows one to make some salient points succinctly. In addition to studies of middlemen like RW, examples include models of monetary exchange (e.g., Kiyotaki and Wright 1993), banking (e.g., Cavalcanti and Wallace 1999), OTC financial markets (e.g., Duffie et al. 2005), unemployment (e.g., Pissarides 2000) and partnership formation (e.g., Burdett and Coles 1997). Still, we go beyond $\{0,1\}$ to see if our main results hinge on it; they do not.

${ }^{4}$ Here we say more about the literature, although it can be skipped if one prefers to see the theory first. Related papers include Bigalser (1993), Wright (1995), Li (1998), Camera (2001), Johri and Leach (2002), Shevchenko (2004), Smith (2004), Masters (2007,2008), Tse (2009) and Watanabe (2010); see Wright and Wong (2014) for more on this work. In subsequent work, Farboodi et al. $(2017,2018)$ have technically similar models, but the applications are quite different. See Hugonnier et al. (2019) and references therein for more on OTC market intermediation. As a referee suggested, we mention that in those models one's buyer/seller status changes over time - one can buy a house, car, bond... and sell it later. We instead have permanent buyer/seller types, as in RW, but changing that may be interesting in future work. It was also suggested that we put up front a comparison to the monetary theory surveyed by Lagos et al. (2017) or Nosal and Rocheteau (2017). Those models have multiplicities ostensibly related to ours, but the mechanism is actually different. There, a seller's decision to accept an asset in exchange depends on what others accept; here the crucial element is strategic interaction in middlemen's decision to adopt buy-and-hold or buy-and-trade strategies. Also, our effect requires endogenous market composition, which is not the case in monetary theory.
} 


\section{Environment}

There is a continuum of infinitely-lived agents of different types. Measure $n_{c}$ of them are end users, or consumers, labeled $C$. The rest choose to be producers, middlemen or nonparticipants, labeled $P, M$ or $N$, with measure $n_{p}, n_{m}$ or $n_{n}$. As discussed below, the market is active if parameters are such that type $P$ produce, in which case they trade with $C$, and might trade with $M$, when they meet. They meet bilaterally in continuous time, with the Poisson rate at which any type $i$ meets type $j$ given by $\alpha_{i j}=\alpha n_{j} / \Sigma_{h} n_{h}$. Note that this displays constant returns since, e.g., doubling $n_{j} \forall j$ doubles the number of meetings and leaves $\alpha_{i j}$ the same. To ease notation, we normalize $n_{c}+n_{p}+n_{m}+n_{n}=1$ and $\alpha=1$, with no loss of generality, and write $\mathbf{n}=\left(n_{c}, n_{p}, n_{m}, n_{n}\right)$.

There are two tradeable objects: $x$, which is indivisible and storable; and $y$, which is divisible but not storable. Type $P$ produces $x$ at cost 0 , which for our applications is without loss of generality. Type $M$ does not produce but can acquire $x$ from $P$. Type $C$ get payoff $u$ from acquiring $x$, which can be interpreted as them consuming it in markets for goods or holding/investing it in markets for assets. Under either interpretation, $P$ and $M$ can hold $x$, but only 0 or 1 unit at a time (this is relaxed below). The costs of holding/storing $x$ for $P$ and $M$ are $\gamma_{p}$ and $\gamma_{m}$, which are positive in markets for goods and negative in markets for assets. In the latter case we use $\rho_{m}=-\gamma_{m}>0$ to denote the flow return on asset holdings. The other tradeable object $y$ is used as a payment instrument when acquiring $x$. It can be produced by anyone at unit cost and consumed by anyone for utility $U(y)$. As a benchmark we set $U(y)=y$, which means transferable utility; the general case is studied in the Appendix.

Type $P$ agents always have 1 unit of $x$, while $M$ can have 0 or 1 in inventory. Let $\mu$ be the fraction of $M$ holding $x$. This increases at rate $n_{p} n_{m}(1-\mu)$ (the measure of $P$ meeting $M$ without $x$ ) and decreases at rate $n_{c} n_{m} \mu \tau$, where $\tau$ is the probability $M$ trades $x$ to $C$ (the measure of $C$ meeting $M$ with $x$ and trading). 
Hence $\dot{\mu}=n_{p} n_{m}(1-\mu)-n_{c} n_{m} \mu \tau$, and in steady state ${ }^{5}$

$$
\mu=\frac{n_{p}}{n_{p}+n_{c} \tau} .
$$

For now we focus on steady states; dynamics are studied in Section 7 .

Bargaining determines the terms of trade: agents $i$ and $j$ split the total surplus with $\theta_{i j}$ denoting the share (bargaining power) of $i$, and $\theta_{j i}=1-\theta_{i j}$, as follows from various common solution concepts (e.g., Nash or Kalai bargaining). Hence when they trade $C$ pays $y_{c p}$ or $y_{c m}$ to $P$ or $M$, while $M$ pays $y_{m p}$ to $P$. The surplus when $C$ trades with $P$, e.g., is $u-y_{c p}=\theta_{c p} u$, since $y_{c p}=\theta_{p c} u$, given that for both the continuation values and outside options cancel. ${ }^{6}$ To keep track of payments, let $\mathbf{y}=\left(y_{c p}, y_{m p}, y_{c m}\right)$.

Next let $V_{p}$ be $P$ 's value function, let $V_{0}$ or $V_{1}$ be $M$ 's value function when he has 0 or 1 unit of $x$, let $V_{c}$ and $V_{n}=0$ be $C$ 's and $N$ 's value functions, and let $\mathbf{V}=\left(V_{p}, V_{0}, V_{1}, V_{c}, V_{n}\right)$. Eliminating the $y$ 's from the $V$ 's using the bargaining solution, we get standard dynamic programming equations

$$
\begin{aligned}
& r V_{p}=n_{c} \theta_{p c} u+n_{m}(1-\mu) \theta_{p m}\left(V_{1}-V_{0}\right)-\gamma_{p}+\dot{V}_{p} \\
& r V_{0}=n_{p} \theta_{m p}\left(V_{1}-V_{0}\right)+\dot{V}_{0} \\
& r V_{1}=n_{c} \tau \theta_{m c}\left(u+V_{0}-V_{1}\right)-\gamma_{m}+\dot{V}_{1} \\
& r V_{c}=n_{p} \tau \theta_{c p} u+n_{m} \mu \theta_{c m}\left(u+V_{0}-V_{1}\right)+\dot{V}_{c},
\end{aligned}
$$

where in steady state $\dot{V}_{j}=0$. In words, (2) says: the flow value $r V_{p}$ is the rate at which $P$ meets $C$ times his share of the surplus; plus the rate at which he meets $M$ without $x$ times his share of that surplus; minus storage cost; plus (out of

\footnotetext{
${ }^{5}$ In deriving (1), we proceed as if $P$ and $M$ trade whenever they meet, which is not valid if $\gamma_{m}>0$ is very big; but in that case $n_{m}=0$, so the conditions are still correct. (Similar rermarks apply to the dynamic programming equations given below.) The situation is simpler when $P$ and $C$ meet, since $C$ always wants $x$ and $P$ can always produce another unit, so they trade. The situation is more complicated when $M$ and $C$ meet, which is why we need $\tau$.

${ }^{6}$ This is because our all agents stay in the market forever, different from the original RW specification, where $M$ stays but $C$ and $P$ exit after one trade; Nosal et al.(2015) argue that having them all stay reduces the algebra without affecting the results too much.
} 
steady state) a capital gain $\dot{V}_{p}$. Similar stories apply to (3)-(5).

Next, for the decision of a nonconsumer to act as type $P$ or $M$, we assume that if he chooses $M$ he starts without $x$ for payoff $V_{0}$ (e.g., if he produced $x$ as a type $P$, he must sacrifice it to acquire the middleman technology). This implies

$$
n_{p}>0 \Rightarrow V_{p} \geq \max \left\{V_{0}, 0\right\} \text { and } n_{m}>0 \Rightarrow V_{0} \geq \max \left\{V_{p}, 0\right\}
$$

and, in particular, $V_{p}=V_{0}$ if $n_{p}, n_{m}>0$. A steady state equilibrium is defined as a nonnegative list $\langle\mu, \mathbf{V}, \mathbf{n}\rangle$ such that: $\mu$ satisfies (1); $\mathbf{V}$ satisfies (2)-(5); and $\mathbf{n}$ satisfies (6). Given $\langle\mu, \mathbf{V}, \mathbf{n}\rangle$ we can compute payments $\mathbf{y}$, the spread $s=y_{c m}-y_{m p}$, the stock of middleman inventories $n_{m} \mu$, etc.

\section{Goods Market Equilibrium}

When $\gamma_{j}>0$ it is immediate that $\tau=1$ ( $M$ with $x$ always trades with $C$ ). Hence there are three possible outcomes. A class 0 equilibrium is one where $n_{p}=n_{m}=0$ and $n_{n}=1-n_{c}$, so the market shuts down. A class 1 equilibrium is one where $n_{p}=1-n_{c}$ and $n_{m}=n_{n}=0$, with production but no intermediation. A class 2 equilibrium is one where $n_{p}>0, n_{m}>0$ and $n_{n}=0$, with production and intermediation. In principle we can have $n_{n}>0, n_{p}>0$ and $n_{m}>0$, too, but it only occurs in a measure 0 set of parameters, so it is ignored. We consider the other possibilities in turn, with detailed proofs in the Appendix.

Consider first class 0 equilibrium, with $n_{p}=n_{m}=0$. This requires $V_{p} \leq 0$ and $V_{0} \leq 0$. When $n_{m}=0, V_{p} \leq 0$ iff $\gamma_{p} \geq \bar{\gamma}_{p} \equiv n_{c} \theta_{p c} u$, and $V_{0}=0$ for all parameters. So class 0 equilibrium exists iff $\gamma_{p} \geq \bar{\gamma}_{p}$, and obviously there are not multiple class 0 equilibria. However, unless parameters satisfy the condition in Lemma 1, a class 0 equilibrium violates subgame perfection and is ignored:

Lemma $1 A$ (subgame perfect) class 0 equilibrium exists iff $\gamma_{p} \geq \bar{\gamma}_{p}$ and $\gamma_{m} \geq$ $g\left(\gamma_{p}\right)$, where $g$ is defined in (9) below. When it exists it is unique. 
Consider next candidate class 1 equilibrium, with $n_{p}=1-n_{c}$ and $n_{m}=0$. This requires $V_{p} \geq 0$ and $V_{p} \geq V_{0}$, so that type $P$ agents do not want to deviate and become type $N$ or $M$. It is easy to check $V_{p} \geq 0$ iff $\gamma_{p} \leq \bar{\gamma}_{p}$, and $V_{p} \geq V_{0}$ iff

$$
\gamma_{m} \geq f\left(\gamma_{p}\right) \equiv \bar{\gamma}_{m}-\frac{r+n_{c} \theta_{m c}+\left(1-n_{c}\right) \theta_{m p}}{\left(1-n_{c}\right) \theta_{m p}}\left(\bar{\gamma}_{p}-\gamma_{p}\right)
$$

where $\bar{\gamma}_{m} \equiv n_{c} \theta_{m c} u$. Since (2)-(5) are linear, there cannot be multiple class 1 equilibria.

Lemma 2 A class 1 equilibrium exists iff $\gamma_{p} \leq \bar{\gamma}_{p}$ and $\gamma_{m} \geq f\left(\gamma_{p}\right)$, where $f$ is defined in (7). When it exists it is unique.

Consider finally class 2 , with $n_{p}, n_{m}>0$. Here it is convenient to proceed using $\mu$ and later recover $\mathbf{n}$. We need $\mu \in(0, \bar{\mu})$, where $\bar{\mu}=1-n_{c}$. Now routine algebra reduces $V_{p}=V_{0}$ to $Q(\mu)=0$, where

$$
Q(\mu)=\kappa_{1} \mu^{2}+\kappa_{2} \mu+\kappa_{3}
$$

is obtained by replacing $n_{p}$ and $n_{m}$ with their values in terms of $\mu$, and the coefficients are

$$
\begin{aligned}
& \kappa_{1}=\theta_{p m}\left(\bar{\gamma}_{m}-\gamma_{m}\right) \\
& \kappa_{2}=-\left[2\left(1-n_{c}\right) \theta_{p m}+n_{c}\right]\left(\bar{\gamma}_{m}-\gamma_{m}\right)-\left(r+n_{c} \theta_{m c}-n_{c} \theta_{m p}\right)\left(\bar{\gamma}_{p}-\gamma_{p}\right) \\
& \kappa_{3}=\left(1-n_{c}\right) \theta_{p m}\left(\bar{\gamma}_{m}-\gamma_{m}\right)+\left(r+n_{c} \theta_{m c}\right)\left(\bar{\gamma}_{p}-\gamma_{p}\right) .
\end{aligned}
$$

We seek $\mu \in(0, \bar{\mu})$ such that $Q(\mu)=0$ and $V_{0} \geq 0$. Now $V_{0} \geq 0$ iff $\gamma_{m} \leq \bar{\gamma}_{m}$, which implies $\kappa_{1}>0$, and hence $Q(\mu)$ is convex. As shown by the curves $Q_{a}, Q_{b}$ and $Q_{c}$ in the right panel of Fig. 1, there are three ways $Q(\mu)$ can have a solution in $(0, \bar{\mu})$ : (a) one root with $Q(0)<0<Q(\bar{\mu})$; (b) one root with $Q(0)>0>Q(\bar{\mu})$; or (c) two roots. The Appendix rules out cases (a) and (c):

Lemma 3 A class 2 equilibrium exists iff $Q(0)>0>Q(\bar{\mu})$. 

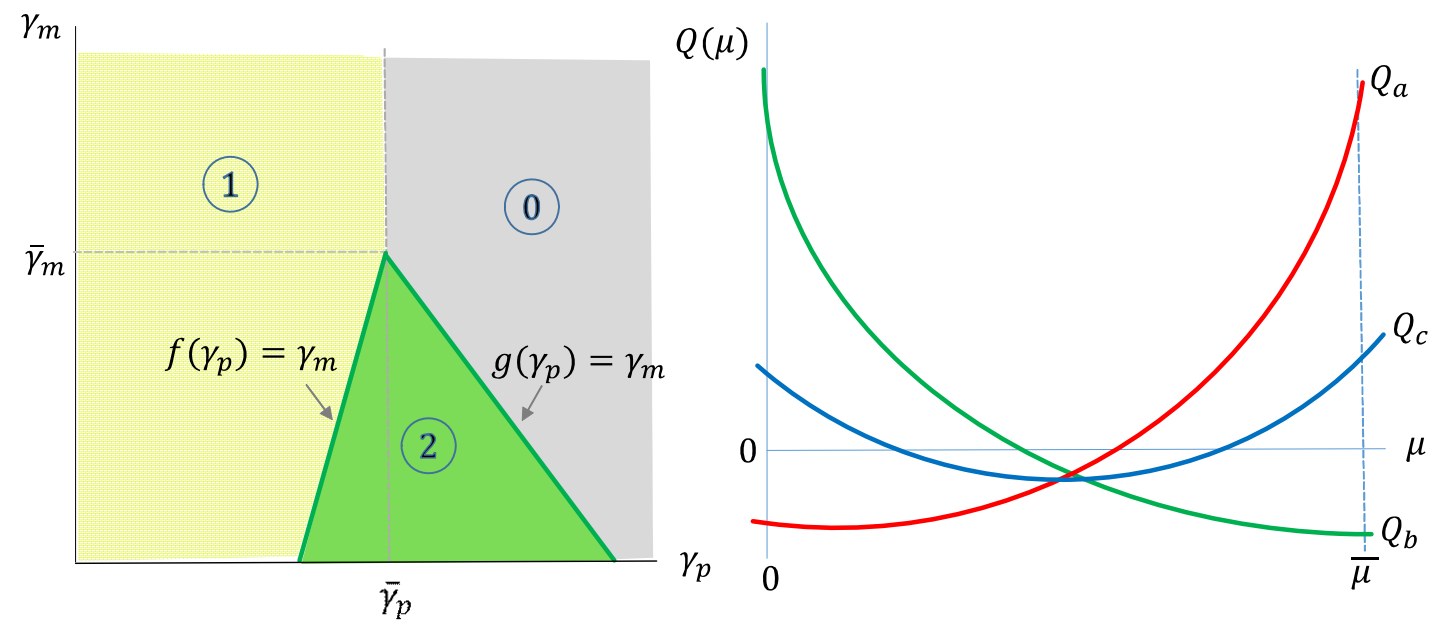

Figure 1: Equilibrium outcomes in $\left(\gamma_{m}, \gamma_{p}\right)$ space

To see when the conditions in Lemma 3 hold, note that $Q(\bar{\mu})<0$ iff $\gamma_{m}<$ $f\left(\gamma_{p}\right)$ where $f$ is defined above, while $Q(0)>0$ iff $\gamma_{m}<g\left(\gamma_{p}\right)$ where

$$
g\left(\gamma_{p}\right) \equiv \bar{\gamma}_{m}+\frac{r+n_{c} \theta_{m c}}{\left(1-n_{c}\right) \theta_{p m}}\left(\bar{\gamma}_{p}-\gamma_{p}\right)
$$

Also, since there is exactly one $\mu \in(0, \bar{\mu})$ with $Q(\mu)=0$, and again (2)-(5) are linear, there cannot be multiple class 2 equilibria.

Lemma 4 A class 2 equilibrium exists iff $\gamma_{m}<f\left(\gamma_{p}\right)$ and $\gamma_{m}<g\left(\gamma_{p}\right)$. When it exists it is unique.

The results are shown in the left panel of Fig. 1, drawn with $f(0)<0$, although $f(0)>0$ is also possible. They accord well with intuition - e.g., intermediation requires $\gamma_{m}$ not too high, naturally, but also requires $\gamma_{p}$ neither too high nor too low, since $P$ does not produce when $\gamma_{p}$ is very high and produces but does not need $M$ when $\gamma_{p}$ is very low. The equilibrating force is this: When $n_{p}$ increases, $P$ is less likely to meet $M$, and when he does it is more likely $M$ already has $x$. Hence raising $n_{p}$ lowers $V_{p}$, which is why we get uniqueness.

Intermediation can be essential in the sense used by monetary theorists: an institution like money is said to be essential if the set of outcomes that can 
be supported as equilibria expands when money is introduced. For money or intermediation the concept is nontrivial, since both are inessential in standard general equilibrium theory. Here, in the region where class 2 equilibrium exists with $\gamma_{p}>\bar{\gamma}_{p}$, production depends on middlemen: if we were to eliminate $M$, say by taxation, the market shuts down. There is more to say about efficiency in Section 4, but for now we summarize the above results as follows:

Proposition 1 With $\gamma_{j}>0$ equilibrium exists and is generically unique, as shown in Fig. 1. For some parameters intermediation is essential.

Additional insights come from changing parameters in class 2 equilibrium. To that end the following is useful:

Lemma 5 An increase in $\gamma_{p}$ shifts $Q(\mu)$ down; an increase in $\gamma_{m}$ shifts $Q(\mu)$ down if $\gamma_{p}<\bar{\gamma}_{p}$ and up if $\gamma_{p}>\bar{\gamma}_{p}$

Based on this it is immediate that

$$
\frac{\partial \mu}{\partial \gamma_{p}}<0, \frac{\partial n_{p}}{\partial \gamma_{p}}<0 \text { and } \frac{\partial n_{m}}{\partial \gamma_{p}}>0
$$

This accords well with intuition: if $\gamma_{p}$ is higher, we get fewer producers, and so middlemen hold $x$ with lower probability. Less intuitive is this:

$$
\begin{aligned}
& \gamma_{p}<\bar{\gamma}_{p} \Rightarrow \frac{\partial \mu}{\partial \gamma_{m}}>0, \frac{\partial n_{p}}{\partial \gamma_{m}}>0 \text { and } \frac{\partial n_{m}}{\partial \gamma_{m}}<0 \\
& \gamma_{p}>\bar{\gamma}_{p} \Rightarrow \frac{\partial \mu}{\partial \gamma_{m}}<0, \frac{\partial n_{p}}{\partial \gamma_{m}}<0 \text { and } \frac{\partial n_{m}}{\partial \gamma_{m}}>0
\end{aligned}
$$

The case $\gamma_{p}>\bar{\gamma}_{p}$ is surprising: how can we get more middlemen when $\gamma_{m}$ is higher? This is answered in Section $4 .^{7}$

\footnotetext{
${ }^{7}$ Among other comparative statics, we showed that increases in $\theta_{m c}$ work like decreases in $\gamma_{m}$, as both make intermediation more profitable, with $\gamma_{m}$ operating in the search process and $\theta_{m c}$ in the bargaining process. We also worked out the effects of demand on the intensive margin (changes in $u$ ) and extensive margin (changes in $n_{c}$ ), calculated the effects of parameters on $\mathbf{y}$ and $s$, etc. but omit the results in the interest of space.
} 


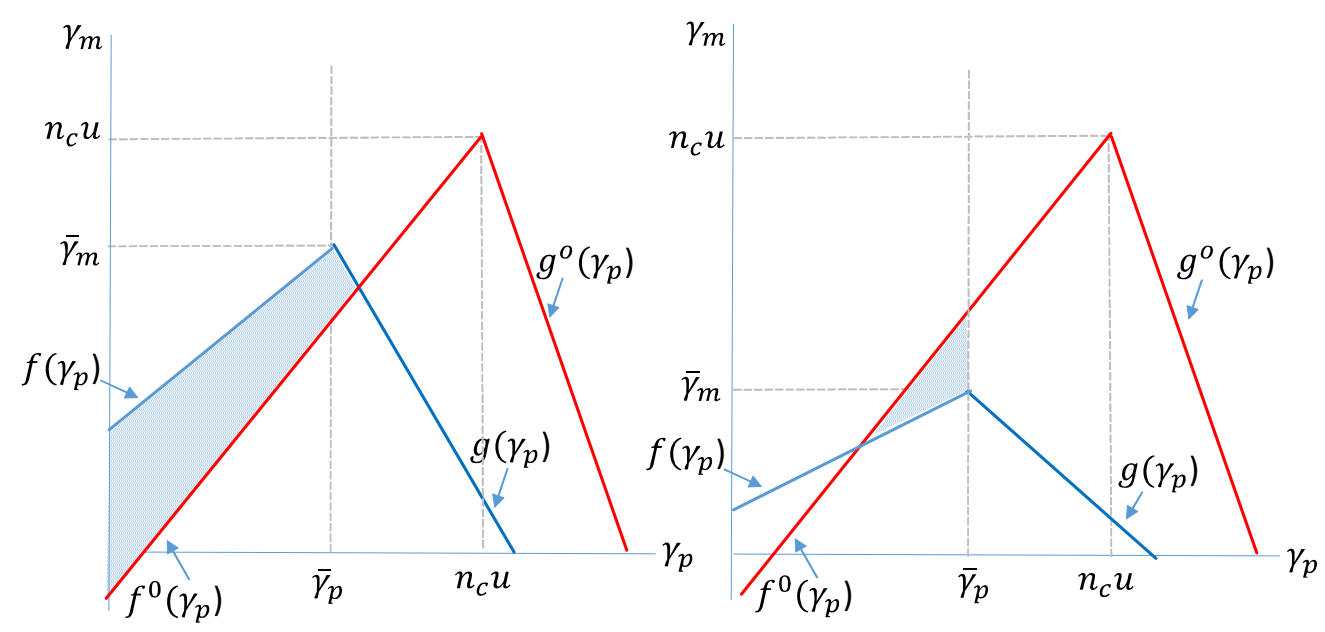

Figure 2: Comparing equilibrium and efficient outcomes

\section{Goods Market Efficiency}

Consider the planner problem with objective function

$$
r W=\max _{\mu, n_{p}, n_{m}} n_{p}\left(n_{c} u-\gamma_{p}\right)+\mu n_{m}\left(n_{c} u-\gamma_{m}\right) \text { st } n_{m}+n_{m}=1-n_{c},
$$

where the first (second) term is the gain from direct (indirect) trade. ${ }^{8}$ The Appendix shows:

Proposition 2 The efficient outcome exists and is generically unique. It has $n_{p}^{o}=n_{m}^{o}=0$ iff $\gamma_{p} \geq n_{c} u$ and $\gamma_{m} \geq g^{o}\left(\gamma_{p}\right) ; n_{p}^{o}>0=n_{m}^{o}$ iff $\gamma_{p} \leq n_{c} u$ and $\gamma_{m} \geq f^{o}\left(\gamma_{p}\right)$; and $n_{p}^{o}, n_{m}^{o}>0$ iff $\gamma_{m}<g^{o}\left(\gamma_{p}\right), f^{o}\left(\gamma_{p}\right)$; where

$$
g^{o}\left(\gamma_{p}\right) \equiv \frac{n_{c}\left(u-\gamma_{p}\right)}{1-n_{c}} \text { and } f^{o}\left(\gamma_{p}\right) \equiv \frac{-n_{c}^{2} u+\gamma_{p}}{1-n_{c}}
$$

Fig. 2 shows $f^{o}$ and $g^{o}$, as well as the analogs from the equilibrium analysis $f$ and $g$, for two examples. Notice the region where $n_{m}^{o}>0$ lies strictly below the $45^{\circ}$ line, so for intermediation to be optimal we need $\gamma_{m}<\gamma_{p}$. In contrast, $\gamma_{m}<\gamma_{p}$

\footnotetext{
${ }^{8}$ Note that (10) can be obtained by summing payoffs over agents in steady state, but maximizing it is equivalent to solving the dynamic planner problem and then letting $r \rightarrow 0$ (see Nosal et al. 2015 for a discussion in a related model).
} 
may or may not entail $n_{m}>0$ in equilibrium, since the decision to become a middlemen depends on the ability to extract rents, captured by bargaining power. Perhaps more importantly, from these examples we know that equilibrium can have too many or too few middleman: equilibrium can have $n_{m}>0$ when the planner wants $n_{m}=0$ or vice versa; and even when the equilibrium and optimum both have middlemen, we do not get $n_{m}=n_{m}^{o}$ in general.

Unsurprisinly, the outcome depends on the $\theta$ 's. First we need $\theta_{p c}^{o}=1$ and $\theta_{m c}^{o}=1$ to avoid holdup problems associated with the costs $\gamma_{p}$ and $\gamma_{m}$, which are sunk when $P$ and $M$ meet $C$. For $\theta_{p m}^{o}$ there is also a holdup problem when $P$ meets $M$, but now other forces arise. One is this: when agents act as type $P$ they neglect making it harder for other $P$ 's to meet $M$ 's and easier for $M$ 's to meet $P$ 's, as usual in search theory. Another, more novel, force is this: higher $n_{p}$ implies higher $\mu$, making it harder for $P$ to trade when they meet $M$. Balancing these forces leads to a version of efficiency results going back to Mortensen (1982) and Hosios (1990) applicable to our three-sided market.

Proposition 3 Let $S_{0}^{o}$, $S_{1}^{o}$ and $S_{2}^{o}$ be the sets of $\gamma^{\prime}$ 's where the efficient outcome is class 0, class 1 and class 2, resp. Equilibrium is efficient iff $\theta_{p c}^{o}=\theta_{m c}^{o}=1$ and: (i) $\left(\gamma_{p}, \gamma_{m}\right) \in S_{0}^{o} \Rightarrow \theta_{p m}^{o}=1$; (ii) $\left(\gamma_{p}, \gamma_{m}\right) \in S_{1}^{o} \Rightarrow \theta_{p m}^{o}=0$; and (iii) $\left(\gamma_{p}, \gamma_{m}\right) \in S_{2}^{o} \Rightarrow$

$$
\theta_{p m}^{o}=\frac{\left(1-\mu^{o}\right)\left(1-n_{c}-\mu^{o}\right)}{\left(1-\mu^{o}\right)\left(1-n_{c}-\mu^{o}\right)+\mu^{o} n_{c}\left[1-\left(n_{c} u-\gamma_{p}\right) /\left(n_{c} u-\gamma_{m}\right)\right]} \in(0,1) .
$$

Next consider how the optimum varies with $\gamma_{j}$. Similar to equilibrium, we have the natural results $\partial \mu^{o} / \partial \gamma_{p}<0, \partial n_{p}^{o} / \partial \gamma_{p}<0$ and $\partial n_{m}^{o} / \partial \gamma_{p}>0$, plus the surprising results

$$
\begin{aligned}
& \gamma_{p}<n_{c} u \Rightarrow \frac{\partial \mu^{o}}{\partial \gamma_{m}}>0, \frac{\partial n_{p}^{o}}{\partial \gamma_{m}}>0 \text { and } \frac{\partial n_{m}^{o}}{\partial \gamma_{m}}<0 \\
& \gamma_{p}>n_{c} u \Rightarrow \frac{\partial \mu^{o}}{\partial \gamma_{m}}<0, \frac{\partial n_{p}^{o}}{\partial \gamma_{m}}<0 \text { and } \frac{\partial n_{m}^{o}}{\partial \gamma_{m}}>0 .
\end{aligned}
$$


How can higher $\gamma_{m}$ lead to more middlemen? To answer that we use this:

Lemma 6 For all parameters, $\partial\left(n_{m}^{o} \mu^{o}\right) / \partial \gamma_{m}<0$.

This says that an increase in $\gamma_{m}$ always reduces the stock of inventories held by middlemen, $n_{m}^{o} \mu^{o}$, but there are two ways to make that happen. One is to reduce $n_{m}^{o}$, which in steady state means higher $\mu^{o}$; the other is to reduce $\mu^{o}$, which means higher $n_{m}^{o}$. When $\gamma_{p}<n_{c} u$ it is optimal to use the extensive margin and reduce $n_{m}^{o}$; when $\gamma_{p}>n_{c} u$ it is optimal to use the intensive margin and reduce $\mu^{o}$, which means higher $n_{m}^{o}$. This explains the planner's choices; the idea is similar for equilibrium.

\section{Asset Market Equilibrium}

Suppose now that storing $x$ is profitable. As a colorful example, suppose $P$ is a painter, $C$ is a collector and $M$ is an art dealer, and that $\rho_{j}=-\gamma_{j}>0$ because paintings generate payoffs simply from gazing at them, or from charging admission to $M$ 's gallery. Will $M$ trade $x$ to $C$ ? Of course the answer depends on fundamentals - i.e., who values it more - but also on the ease or difficulty with which inventory can be replaced and that depends on equilibrium considerations. Indeed, one might say that liquidity is a key factor: the market can be said to be more liquid when it is easier to get $x$, which is the case when $n_{p}$ is bigger, which is the case when $\tau$ is bigger, because that makes $V_{p}$ higher.

This is relevant for any asset with positive net returns, not only art. As a financial application, suppose $P$ produces or otherwise obtains capital suitable for investment by either $C$ or $M$. Then a type $M$ agent with capital may not want to pass it on to $C$, again depending on fundamentals like $\rho_{m}$, but also on the strategies of others. This interpretation allows us to investigate the view (recall fn.2) that financial institutions may be more unstable/fragile that other intermediaries, such as those in retail goods markets. 
As another application, $x$ can be a interpreted as housing that provides utility as shelter, in which case $M$ must decide whether to keep it as a residence or flip it to $C$. With this interpretation we can investigate the idea that real estate markets are susceptible to "hot and cold spells" depending on the speculations of flippers. Of course, the model is quite abstract, but we think it still provides insights into these kinds of applications. Moreover, our position is not that the only relevant distinction between goods or assets is $\gamma_{m}>0$ or $\gamma_{m}<0$; our position is that it is a distinction that is interesting because of the way it affects results. ${ }^{9}$

While $\tau=1$ is immediate for $\gamma_{m}>0$, it is not for $\gamma_{m}<0$, and so there are more candidate equilibria. We call $n_{m}=0$ and $\tau=1$ a class $1^{T}$ equilibrium, with $T$ indicating $M$ trades $x$ to $C$ (in fact there are no type $M$ agents on the equilibrium path, but off this path type $M$ with $x$ would trade it to $C$ ). Similarly, $n_{m}=0$ and $\tau=0$ is a class $1^{K}$ equilibrium, with $K$ indicating $M$ keeps $x$. Also, $n_{m}=0$ and $\tau \in(0,1)$ is a class $1^{R}$ equilibrium, with $R$ indicating $M$ randomizes, but we ignore it because it can only be an equilibrium for nongeneric parameters. We also ignore $n_{m}=1-n_{c}$, which is uninteresting, because there is no production, and unnecessary for our purposes. Thus, in addition to $1^{K}$ and $1^{T}$, the other relevant candidates all have $n_{m} \in\left(0,1-n_{c}\right)$ and either: $\tau=1$, which is a class $2^{T}$ equilibrium; $\tau=0$, which is a class $2^{K}$ equilibrium; or $\tau \in(0,1)$, which is a class $2^{R}$ equilibrium. See Table 1 .

\begin{tabular}{|c||c|c|c|}
\hline$n_{m} \backslash \tau$ & 0 & {$[0,1]$} & 1 \\
\hline \hline 0 & $1^{K}$ & $\times$ & $1^{T}$ \\
\hline$\left(0,1-n_{c}\right)$ & $2^{K}$ & $2^{R}$ & $2^{T}$ \\
\hline $1-n_{c}$ & $\times$ & $\times$ & $\times$ \\
\hline
\end{tabular}

Table 1: Candidate equilibria with $\rho_{j}=-\gamma_{j}>0$.

\footnotetext{
${ }^{9}$ It is clear that what matters is $\rho_{m}>0$, not $\rho_{p}>0$, but for symmetry here we assume both. Also, an editor suggested we mention that in reality there are primary and secondary asset markets: the former have buyers trading with originators, captured by $P$ in the model, while the latter have them trading with others who previously bought the asset, captured by $M$. While some assets trade exclusively or mainly in secondary markets, we let $C$ potentially get $x$ from either $P$ or $M$, the way one can get, e.g., T-Bills directly from the government or indirectly through a security dealer. Gong (2019) analyzes in greater depth a related model that determines endogenously whether $C$ can buy from only $P$, from only $M$, or from both.
} 
Lemma 7 states some results formally, but they are perhaps better understood from Fig. 3, which in one panel has the negative quadrant of $\left(\gamma_{p}, \gamma_{m}\right)$, space and in the other the positive quadrant of $\left(\rho_{p}, \rho_{m}\right)$ space, which we find easier to interpret. While there are various issues that can be discussed using the results - e.g., as in the model with $\gamma_{j}>0$, one can ask about the parameters that are more likely to make intermediation an equilibrium outcome - but we highlight the following: Suppose $\rho_{p}$ is not too high, which is necessary for $n_{m}>0$. Then for $\rho_{m}$ very high we get $\tau=0$ and for $\rho_{m}$ very low we get $\tau=1$, naturally, but for $\rho_{m}$ neither too high nor too low there coexist a $2^{K}$ equilibrium with $\tau=0$, a $2^{T}$ equilibrium with $\tau=1$, and a $2^{R}$ equilibrium with $\tau \in(0,1)$.

\section{Lemma 7 Define}

$$
\begin{aligned}
\widehat{\gamma}_{m} & \equiv-u\left[r+\left(1-n_{c}\right) \theta_{m p}\right] \\
\widehat{f}\left(\gamma_{p}\right) & \equiv-\left(\bar{\gamma}_{p}-\gamma_{p}\right) \frac{r+\left(1-n_{c}\right) \theta_{m p}}{\left(1-n_{c}\right) \theta_{m p}} \\
k\left(\gamma_{p}\right) & \equiv-r u-\bar{\gamma}_{p}+\gamma_{p} .
\end{aligned}
$$

Also define $\widehat{k}\left(\gamma_{p}\right)$ to be the lower root of the quadratic given in the proof in the Appendix. Then class $1^{T}$ equilibrium exists iff $\gamma_{m} \geq \max \left\{\widehat{\gamma}_{m}, f\left(\gamma_{p}\right)\right\}$; class $1^{K}$ exists iff $\widehat{f}\left(\gamma_{p}\right) \leq \gamma_{m} \leq \widehat{\gamma}_{m}$; class $2^{K}$ exists iff $\gamma_{m} \leq \min \left\{k\left(\gamma_{p}\right), \widehat{f}\left(\gamma_{p}\right)\right\} ;$ class $2^{R}$ exists iff $\widehat{k}\left(\gamma_{p}\right)<\gamma_{m}<k\left(\gamma_{p}\right)$; and class $2^{T}$ exists iff $\widehat{k}\left(\gamma_{p}\right) \leq \gamma_{m} \leq f\left(\gamma_{p}\right)$.

We conclude that $\gamma_{j}<0$ implies the liquidity of the market, and in particular whether $x$ is passed on to $C$ or "hoarded" by $M$, can be a self-fulfilling prophecy, i.e., not pinned down by fundamentals. This constitutes part (i) of Proposition 4. Part (ii) says that if $\mathbf{n}$ is fixed we get back uniqueness even with $\gamma_{j}<0$, confirming that multiplicity requires both $\gamma_{j}<0$ and endogenous $n_{p}$.

Proposition 4 (i) With $\gamma_{j}<0$ equilibrium exists. As shown in Fig. 3, $\forall \gamma_{p}<$ $\tilde{\gamma}_{p}$ where $\tilde{\gamma}_{p}<0$, and $\gamma_{m}$ neither too high nor too low class $2^{K}, 2^{T}$ and $2^{R}$ equilibria coexist. (ii) If $n_{m}$ and $n_{p}$ are fixed equilibrium is unique: $\rho_{m}=-\gamma_{m}>$ $\left(r+n_{p} \theta_{m p}\right) u \Rightarrow \tau=0$ and $\rho_{m}<\left(r+n_{p} \theta_{m p}\right) u \Rightarrow \tau=1$. 

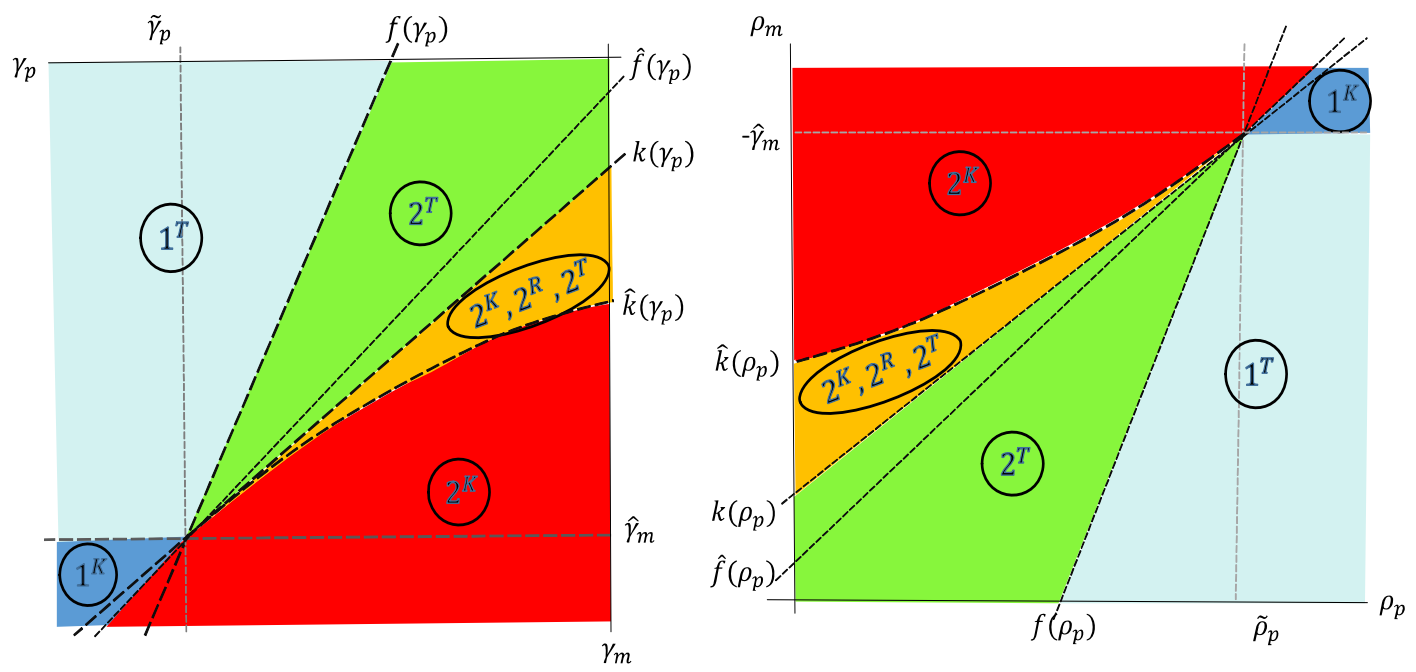

Figure 3: Outcomes in $\left(\gamma_{p}, \gamma_{m}\right)$ and in $\left(\rho_{p}, \rho_{m}\right)$ space

\section{Asset Market Efficiency}

One can emulate the method in Section 4 for the planner, but now we have to choose $\tau$ as well as $n_{m}$, so the details are relegated to a Supplemental Appendix, and here we report just a few findings. ${ }^{10}$ First, depending on parameters $n_{m}$ can be too big or too small, as in the model with $\gamma_{j}>0$. However, different from that model, with $\gamma_{j}<0$ it is not possible to find $\theta$ 's such that equilibrium is efficient even if we select the best equilibrium when there is multiplicity. To see this, note that the Supplemental Appendix shows the borders between regions are the same for the equilibrium and planner iff $\theta_{p c}=\theta_{m c}=\theta_{m p}=1$; but with those $\theta$ 's the values of $n_{m}$ in the $2^{T}$ region are different.

\footnotetext{
${ }^{10}$ The method involves checking each $\left(\tau, n_{m}\right)$ pair in Table 1 to determine the parameters for which it solves the planner problem. To be a solution $\left(\tau, n_{m}\right)$ must satisfy these conditions: a corner solution $\tau=0(\tau=1)$ requires $r W$ decreasing in $\tau$ at $\tau=0$ (increasing in $\tau$ at $\tau=1)$; a corner solution for $n_{m}$ requires something similar; and interior solutions for $\tau \in(0,1)$ or $n_{m} \in\left(0,1-n_{c}\right)$ must satisfy standard FOC's and SOC's. After exhausting the candidates in Table 1, we can partition parameter space into regions as follows: for big $\rho_{p}$ the efficient outcome is $1^{T}$ or $1^{K}$ as $\rho_{m}$ is small or big; for small $\rho_{p}$ it is $2^{T}$ or $2^{K}$ as $\rho_{m}$ is small or big. The result is simple enough, and qualitatively the four outcomes are similar to the four classes of equilibria analyzed above, but the regions where they obtain are different. What is slightly harder is this: in some regions there is more than one $\left(\tau, n_{m}\right)$ satisfying the FOC's, so we have to check the SOC's, and also compare $r W$ across local maximizers to find the global maximizer. See the Supplemental Appendix.
} 
While in equilibrium $n_{m}$ can be too big or too small, there is no analogous result for $\tau$. It is easy to show $\tau$ can be too small - i.e., we can have $\tau=0$ in equilibrium when the planner wants $\tau=1$ - but we did not find an example where $\tau=1$ in equilibrium when the planner wants $\tau=0$ (although we did not prove it is impossible). We understand the situation in terms of coordination. When $\tau=0, M$ is willing to sell $x$ to $C$ in principle, but not in practice, because inventories take too long to replace. If every $M$ were to change to $\tau=1$, however, $V_{p}$ and hence $n_{p}$ would rise, and then would $M$ would trade $x$ to $C$ since it would take less time to replace.

Moreover, when equilibria with $\tau=0$ and $\tau=1$ coexist, the latter is better. Although the result does not depend on this, it is easiest to see when $\theta_{p c}=\theta_{m c}=$ 1 , since then welfare is unambiguously measured by $V_{p}=V_{0}$ in any equilibrium with $n_{m}>0 .{ }^{11}$ In this case, note that the two equilibria imply

$$
\begin{aligned}
& r V_{p}(\tau=0)=\left(1-n_{c}\right)\left(n_{c} u-\gamma_{p}\right) \\
& r V_{p}(\tau=1)=\left(1-n_{c}\right)\left[n_{c} u-\gamma_{p}+n_{m}(1-\mu)\left(V_{1}-V_{0}\right)\right]
\end{aligned}
$$

Hence, $r V_{p}(\tau=1)>r V_{p}(\tau=0)$, consistent with interpreting $\tau=0$ as a coordination problem. We summarize as follows:

Proposition 5 Similar to goods markets, in asset markets the efficient outcome exists and is generically unique, and equilibrium can have $n_{m}$ too big or too small. Different from goods markets, we cannot set the $\theta$ 's so that equilibrium is efficient. Also, when equilibria with $\tau=0$ and $\tau=1$ coexist the latter is better.

\section{Asset Market Dynamics}

It is convenient here to work in $\left(n_{1}, \Delta\right)$ space, where $n_{1}=n_{m} \mu$ is the measure of inventories held by $M$, which is predetermined at any point in time $t$, while $\Delta=$

\footnotetext{
${ }^{11}$ Coexistence requires $n_{m}>0$, which requires $V_{p}=V_{0}$, and when $\theta_{p c}=\theta_{m c}=1$ all the surplus goes to nonconsumers. When $\theta_{p c}<1$ or $\theta_{m c}<1$ consumers get some surplus, too, but the result goes through if we define welfare by $r W$, which aggregates $r V_{c}$ and $r V_{p}$.
} 
$V_{1}-V_{0}$ represents (beliefs about) the value of inventories. Then the equilibrium conditions can be collapsed to a two-dimensional dynamical system in $\left(n_{1}, \Delta\right)$. For $\gamma_{j}>0$, the Appendix shows the unique steady state is a saddle point: for any initial condition $n_{1}=\bar{n}_{1}$ there is a unique $\bar{\Delta}$ such that starting at $\left(\bar{n}_{1}, \bar{\Delta}\right)$ the system transits to steady state; and for any other $\bar{\Delta}$ the system follows an explosive path. In other words, when $\gamma_{j}>0$ equilibrium, not only steady state, is unique.

For $\gamma_{j}<0$, the dynamics are more complicated. ${ }^{12}$ To begin, as in the steady state analysis, let us assume type $P$ can at any $t$ become type $M$, but must start with 0 inventory. Next, since type $C$ agents really make no decisions, we can ignore them and focus on

$$
\begin{aligned}
& r V_{p}=n_{c} \theta_{p c} u+n_{0} \theta_{p m} \Delta-\gamma_{p}+\dot{V}_{p} \\
& r V_{0}=\left(1-n_{c}-n_{1}-n_{0}\right) \theta_{m p} \Delta+\dot{V}_{0} \\
& r V_{1}=n_{c} \tau \theta_{m c}(u-\Delta)-\gamma_{m}+\dot{V}_{1} .
\end{aligned}
$$

The interesting case concerns $n_{m}>0$, where $V_{p}=V_{0}$. This holds iff $n_{0}=$ $n_{0}\left(n_{1}, \Delta\right)$, where we can solve explicitly for

$$
n_{0}\left(n_{1}, \Delta\right)=\frac{\gamma_{p}-n_{c} \theta_{p c} u}{\Delta}+\left(1-n_{c}-n_{1}\right) \theta_{m p} .
$$

Next, subtracting (16)-(17), we get

$$
\dot{\Delta}=r \Delta-n_{c} \tau \theta_{m c}(u-\Delta)+\gamma_{m}+\left(1-n_{c}-n_{1}-n_{0}\right) \theta_{m p} \Delta .
$$

Now $\tau=\tau(\Delta)$ is $1,[0,1]$ or 0 as $\Delta-u$ is positive, 0 or negative, which we can insert along with $n_{0}\left(n_{1}, \Delta\right)$ into (18) to arrive at

$$
\dot{\Delta}=r \Delta-n_{c} \tau(\Delta) \theta_{m c}(u-\Delta)+\gamma_{m}+\left[1-n_{c}-n_{1}-n_{0}\left(n_{1}, \Delta\right)\right] \theta_{m p} \Delta .
$$

\footnotetext{
${ }^{12}$ While some previous search models also display interesting dynamics - e.g., Diamond and Fudenberg (1989), Boldrin et al. (1993) or Mortensen (1999) - they hinge crucially on increasing returns in the matching or production technology; that is not the case here.
} 

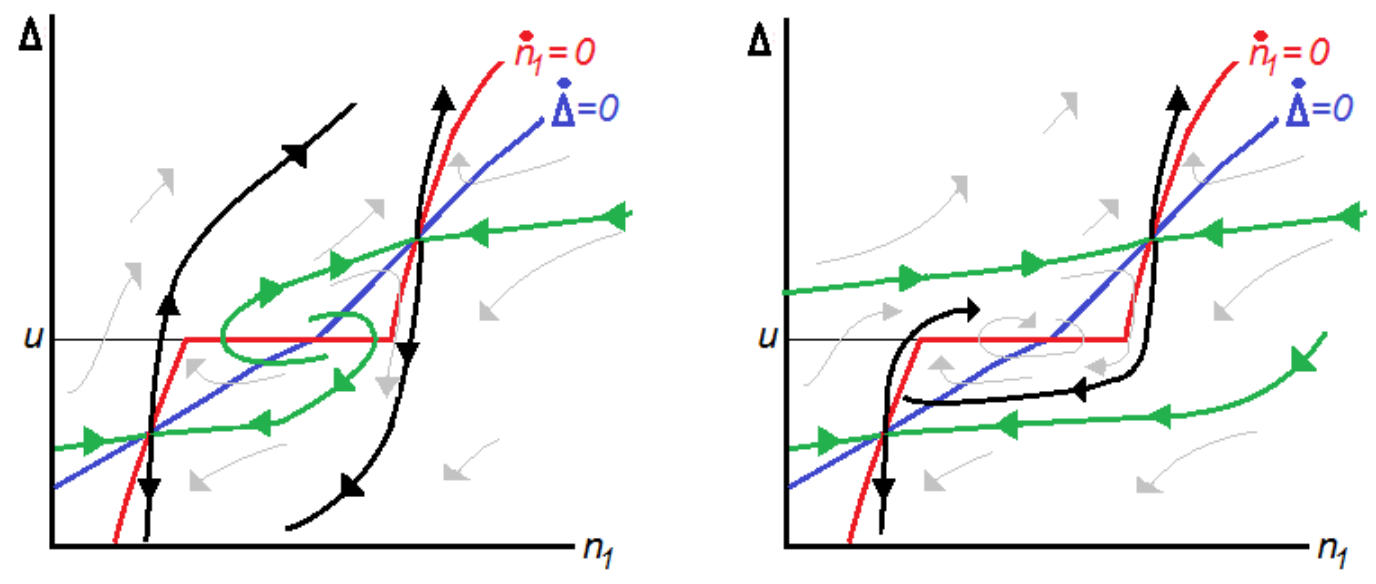

Figure 4: The phase plane with $\rho_{j}>0$

Thus we get a differential equation for $\Delta$. The equation for $n_{1}$ is

$$
\dot{n}_{1}=n_{0}\left(n_{1}, \Delta\right)\left[1-n_{c}-n_{1}-n_{0}\left(n_{1}, \Delta\right)\right]-n_{1} n_{c} \tau(\Delta) .
$$

Then, as is standard, given an initial $\bar{n}_{1}$ equilibrium defined as a bounded and nonnegative path $\left(n_{1}, \Delta\right)$ satisfying (19)-(20) (boundedness comes from the transversality condition; see, e.g., Rocheteau and Wright 2013).

We know that for some parameters there are 3 steady states, as shown in Fig. 4 . Notice in this phase plane the $\dot{n}_{1}=0$ curve has a flat spot and the $\dot{\Delta}=0$ curve has a kink at $\Delta=u$, which is where $\tau$ switches from 0 to 1 . The lower steady state, $\left(n_{1}^{L}, \Delta^{L}\right)$, has $\Delta<u$ and $\tau=1$; the higher one, $\left(n_{1}^{H}, \Delta^{H}\right)$, has $\Delta>u$ and $\tau=0$; and the middle one $\left(n_{1}^{M}, \Delta^{M}\right)$ has $\Delta=u$ and $\tau \in(0,1)$. One can check that $\left(n_{1}^{L}, \Delta^{L}\right)$ and $\left(n_{1}^{H}, \Delta^{H}\right)$ are saddle points, but the dynamics around $\left(n_{1}^{M}, \Delta^{M}\right)$ can be complicated.

To see this, consider an example with $u=1, r=0.05, n_{c}=0.3, \theta_{p m}=0.5$, $\theta_{p c}=\theta_{m c}=1, \rho_{m}=0.36$ and $\rho_{p}=0$. As shown in the upper left panel of Fig. 5 , the three steady states are approximately $n_{1}^{L}=0.05, n_{1}^{M}=0.07$ and $n_{1}^{H}=0.25$. The upper right panel zooms in to show local dynamics around $\left(n_{1}^{M}, \Delta^{M}\right)$. Whether this converges to steady state, or to a small cycle around it, 

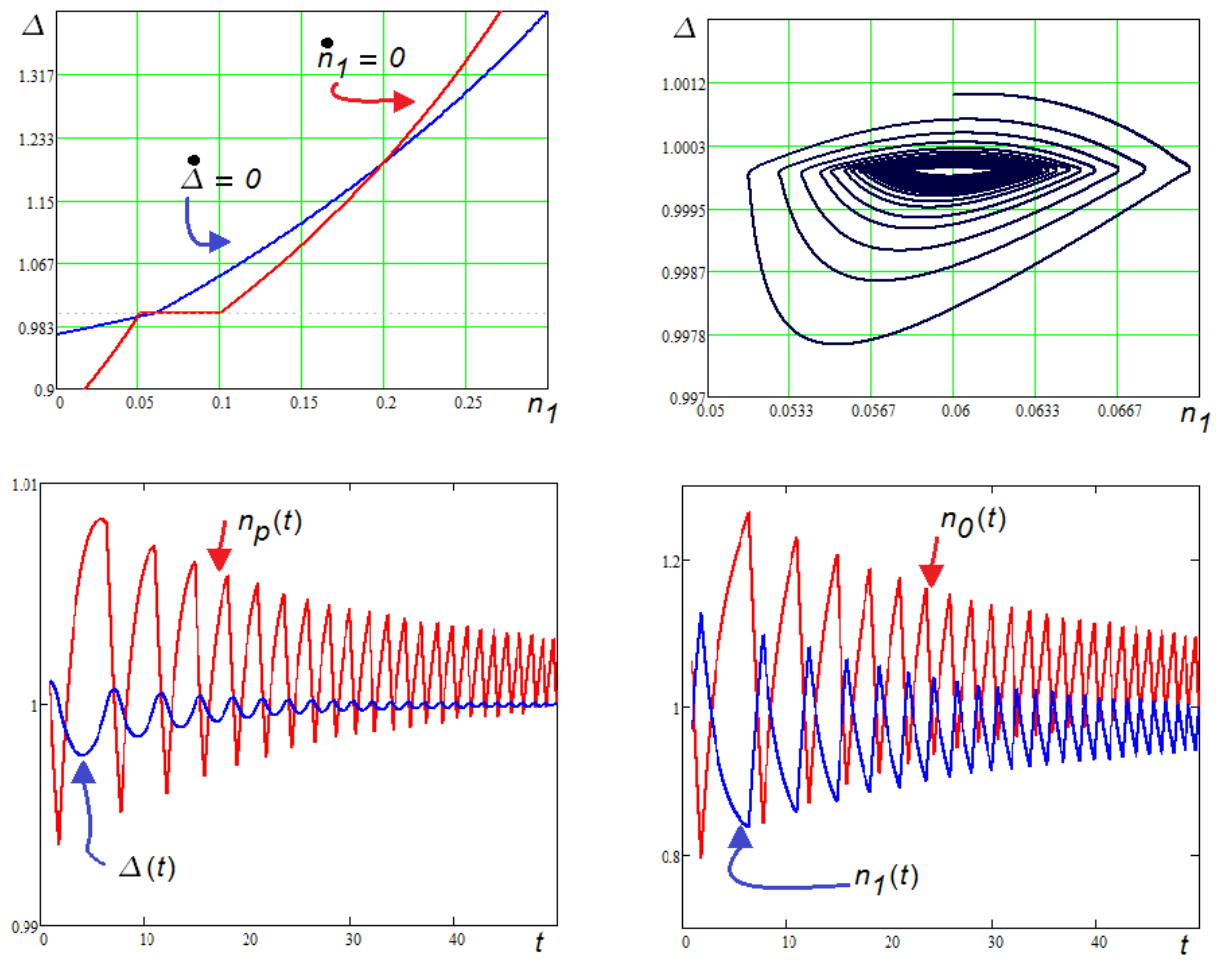

Figure 5: Dynamics in an example with $\rho_{m}>0$

is hard to say from the numerical output, and checking local stability directly is hindered by the $\dot{n}_{1}=0$ curve being nondifferentiable at $\Delta=u$. But we can learn a lot numerically. Figure 6 shows the time path of $n_{1}$ in the example perturbed by reducing $\theta_{p c}$ to 0.1 , starting with $n_{1}$ close to $n_{1}^{M}$. Clearly, these equilibrium fluctuations do not settle down, and they not small.

More generally, starting with any $\bar{n}_{1}$ in the neighborhood of $n_{1}^{M}$, there is a continuum of equilibria indexed the choice of $\bar{\Delta}$ over some range, because all paths starting at $\left(\bar{n}_{1}, \bar{\Delta}\right)$ are bounded and nonnegative. After a shock to the system - e.g., an unexpected drop in $\mu$ for whatever reason - there are many equilibria that cycle around $\left(n_{1}^{M}, \Delta^{M}\right)$ as in Fig. 5 or 6 . Hence, small changes in fundamentals can generate very volatile reactions. Also notice that while the fluctuations in $\Delta$ or $n_{p}$ are not that big relative to their long-run averages, the fluctuations in $n_{1}$ and $n_{0}$ are around $10 \%$ and $20 \%$, so while this is not a serious 


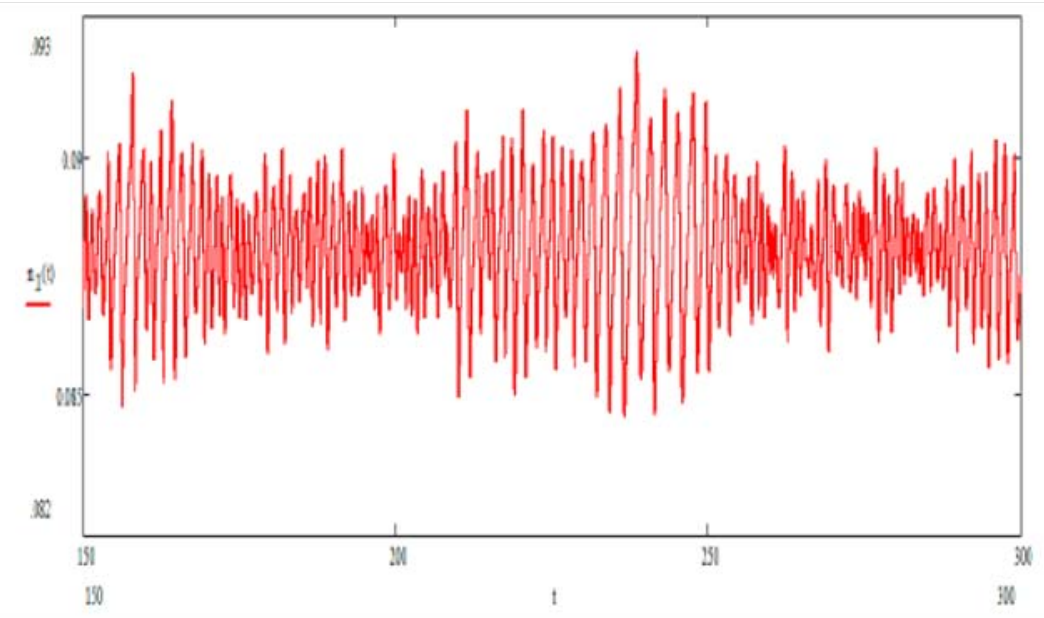

Figure 6: Persistent fluctuations in equilibrium

calibration, it is interesting that fluctuations in inventories are much bigger than output, a stylized fact about business cycles.

Also, in Fig. 4, the left panel shows the stable manifolds of the two saddle points trapped inside the unstable manifolds. This means the stable manifolds must wrap around the middle steady state, either emanating from it or from a cycle around it. In either case, starting with any $\bar{n}_{1}$ in the neighborhood of $n_{1}^{M}$, there is a continuum of equilibria indexed by $\bar{\Delta}$, and after a shock there are many different equilibrium paths that fluctuate around $\left(n_{1}^{M}, \Delta^{M}\right)$, as well as paths starting on one of the stable manifolds and asymtoptically approaching either $\left(n_{1}^{L}, \Delta^{L}\right)$ or $\left(n_{1}^{H}, \Delta^{H}\right)$. The right panel is similar, except that for any $\bar{n}_{1}$ in a large range, depending on initial beliefs, the system can transit to $\left(n_{1}^{L}, \Delta^{L}\right)$ or $\left(n_{1}^{H}, \Delta^{H}\right)$, or oscillate on its way to $\left(n_{1}^{M}, \Delta^{M}\right)$ or a cycle around it. ${ }^{13}$

\footnotetext{
${ }^{13}$ One can also construct stochastic (sunspot) equilibria using standard methods. Consider a random variable $\sigma$ that affects nothing fundamental but could affect behavior if agents believe it will. According to a Poisson process $\sigma$ switches from $\sigma_{1}$ to $\sigma_{2}$ at rate $\eta_{1}$ and switches back at rate $\eta_{2}$, and at each switch the economy jumps from one (bounded) trajectory in Fig. 4 to another. Now given rational expectations about the jumps, Fig. 4 would actually change, but it is qualitatively similar if the $\eta$ 's are not too big. In particular, when the phase plane looks like the right panel, one equilibrium has the economy jumping between paths approximately given by the stable manifolds of the steady states with $\tau=1$ and $\tau=0$, with $M$ 's behavior and the direction of the inventory path switching whenever $\Delta$ crosses $u$. For more discussion see, e.g., Kaplan and Menzio (2016) who study different but related kinds of models.
} 
Thus our intermediated asset market not only has multiple steady states, but dynamic indeterminacy and excess volatility (fluctuations in endogenous variables when fundamentals are constant). It is also subject to fragility, where a small change in fundamentals can lead to a structural change in equilibrium. Similar results appear in other models, but the economics is different, arising here from complementarities in trading strategies and endogenous market composition.

\section{Robustness}

The results are robust on several dimensions. First, in the Appendix we extend the uniqueness result for $\gamma_{j}>0$ to the case where we give multiplicity a fighting chance by going beyond linear utility and steady state. This is relevant because some search models display multiplicity if one considers nonlinear utility or dynamics, but not if one imposes linearity and looks only at steady state (e.g., Wright and Wong 2014; Trejos and Wright 2016); that is not the case here.

One commentator conjectured that treating types $C$ and $M$ asymmetrically might be driving some results. We think our setup is natural, but it is true that: (i) $C$ gets payoff $u$ from $x$ while $M$ gets payoff $\rho_{m} / r$ from $x$; and (ii) after acquiring it $C$ can get another $x$, while $M$ is restricted to $\{0,1\}$. So, consider instead: (i) $C$ gets $\rho_{c} / r$ from $x$; and (ii) $C$ also is restricted to $x \in\{0,1\}$. This makes them symmetric, but without further modification, eventually all end users can end up with $x$ and production shuts down. So, suppose that, when $C$ acquires $x$, he leaves the market to be replaced by a new $C$ (we could also let $x$ depreciate as discussed below).

To ease notation, set $\theta_{p c}=\theta_{m c}=1$ and write

$$
\begin{aligned}
r V_{p} & =n_{c} \rho_{c} / r+n_{m}(1-\mu) \theta_{p m}\left(V_{1}-V_{0}\right)+\rho_{p} \\
r V_{0} & =n_{p} \theta_{m p}\left(V_{1}-V_{0}\right) \\
r V_{1} & =n_{c} \tau\left(\rho_{c} / r+V_{0}-V_{1}\right)+\rho_{m} .
\end{aligned}
$$


Now $\tau=1$ if $\rho_{c} / r+V_{0}-V_{1}>0$, which reduces to

$$
\rho_{c}>\rho^{*} \equiv \frac{r}{r+n_{p} \theta_{m p}} \rho_{m}
$$

Hence, $M$ and $C$ trade when $C^{\prime}$ 's valuation is above $\rho^{*}$, not surprisingly, but there are two points worth mention: first, $n_{p} \theta_{m p}>0$ implies $\rho^{*}<\rho_{m}$, so it is not the case that $M$ trades $x$ to $C$ iff $\rho_{c}>\rho_{m}$; second, $\rho^{*}$ depends on the endogenous $n_{p}$, not merely parameters.

Other commentators conjectured that we do not need type $M$, as similar multiplicities can occur with only $P$ and $C$. That is more delicate. One approach is to eliminate $M$ and let agents choose to be either $C$ or $P$, with $n_{p}+n_{c}=1$. Letting $\tau$ now denote the probability $P$ and $C$ trade, we have

$$
\begin{aligned}
& r V_{c}=n_{p} \tau\left(1-\theta_{p c}\right) u \\
& r V_{p}=\left(1-n_{p}\right) \tau \theta_{p c} u+\rho,
\end{aligned}
$$

It is easy to check that as long as $\theta_{p c} \neq 0$ the unique equilibrium has $\tau=1$. For $\theta_{p c}=0$, multiplicity emerges, because then $r V_{c}=n_{p} \tau u$ and $r V_{p}=\rho$, so we can have $n_{p} \in(0,1)$ as long as $n_{p} \tau u=\rho$, and there are many combinations of $\left(n_{p}, \tau\right)$ satisfying this condition. But that is not interesting, because it only works at $\theta_{p c}=0$, and it is payoff irrelevant. So we do need $M$ in this version.

A better conjecture by one referee is that a similar multiplicity may emerge without $M$ when we give $C$ a trade off between saving $x$ and consuming it. This is plausible, since $C$ consuming $x$ is similar to $M$ trading it away. $o$ pursue it, suppose there are two types, $C$ that can save or consume $x$, and $P$ that can produce it or be a nonparticipant. In the Supplemental Appendix we confirm this works: if $C$ is more likely to consume $x$, there will be more $C$ searching for $x$, which encourages participation by $P$ and makes $C$ more likely to consume. Multiplicity can emerge. This changed our views, given we previously thought middlemen were necessary for this kind of multiplicity; they are not. Yet it does 
not change a main conclusion: in models with middlemen, it is still true that multiplicity and complicated dynamics emerge only if $\gamma_{m}<0$ and only if market composition is endogenous.

Another extension is to let $M$ hold inventories $i \in\{0,1, \ldots I\}$, where $1<I<$ $\infty .{ }^{14}$ Let $\mathbf{n}=\left(n_{0}, n_{1}, \ldots n_{I}\right)$, where $n_{i}$ is the measure of $M$ with $i$ units of $x$. As Fig. 7 shows, $M$ moves up the distribution by one unit at rate $\varepsilon_{U}=n_{P}$, since he trades whenever he meets $P$ (we maintain that $P$ can only produce one unit per meeting). Also, $M$ moves down one unit at rate $\varepsilon_{D}^{i}=n_{C} \tau_{i}+\delta$, where $\tau_{i} \in\{0,1\}$ indicates whether or not $M$ with $i$ units trades with $C$ (we maintain that $C$ only wants one unit per meeting), and each unit depreciates at Poisson rate $\delta$. If $V_{i}$ is the value function for $M$ with $i$, then $\tau_{i}=1$ if $u>\Delta_{i}=V_{i}-V_{i-1}$.
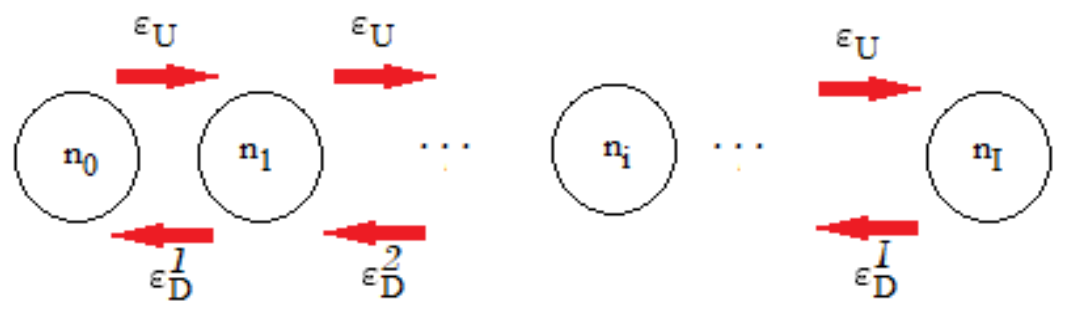

Figure 7: Inventory flows

Now steady state solves

$$
\begin{aligned}
n_{0} n_{p}= & n_{1}\left(\delta+n_{c} \tau_{1}\right) \\
& \cdots \\
n_{i} n_{p}+n_{i}\left(\delta+n_{c} \tau_{i}\right)= & n_{i-1} n_{p}+n_{i+1}\left(\delta+n_{c} \tau_{i+1}\right) \\
& \cdots \\
n_{I}\left(\delta+n_{c} \tau_{I}\right)= & n_{I-1} n_{p}
\end{aligned}
$$

\footnotetext{
${ }^{14}$ One can consider $I=\infty$, too, but we think $I<\infty$ makes sense. The reason is that if there were an increasing marginal cost or decreasing marginal benefit of inventories, and $M$ could choose $I$, he would choose $I<\infty$ (see Shevchenko 2004). Also, for this extension we assume $M$ types never meet each other. With $i \in\{0,1\}$ this does not matter, since there are no gains from trade between $M$ 's, but here it would matter and might be worth studying (see Afonso and Lagos 2015 for something along these lines applied to banks in the Fed Funds market).
} 
The first equation implies $n_{1}=n_{0} n_{p} /\left(\delta+n_{c} \tau_{1}\right)$. Then the second reduces to $n_{1} n_{p}=n_{2}\left(\delta+n_{c} \tau_{2}\right)$, or

$$
n_{2}=\frac{n_{1} n_{p}}{\left(\delta+n_{c} \tau_{2}\right)}=\frac{n_{0} n_{p}^{2}}{\left(\delta+n_{c} \tau_{1}\right)\left(\delta+n_{c} \tau_{2}\right)}
$$

Continuing in this way $\forall i \in\{1,2 \ldots I\}$ we get

$$
n_{i}=\frac{n_{0} n_{p}^{i}}{\prod_{j=1}^{i}\left(\delta+n_{c} \tau_{j}\right)} .
$$

Note the last equation holds automatically when the others hold, so we replace it with $\sum_{i=0}^{I} n_{i}=1-n_{c}-n_{p}$, or,

$$
n_{0}=\frac{1-n_{c}-n_{p}}{1+\sum_{i=1}^{I} \frac{n_{p}^{i}}{\prod_{j=1}^{i}\left(\delta+n_{c} \tau_{j}\right)}} .
$$

This is the closed-form for $\mathbf{n}$ given any $\boldsymbol{\tau}$ and $n_{p} \cdot{ }^{15}$

Let $\rho(i)=i \rho_{M}$ be $M$ 's return from holding $i$ units, with $\rho_{m}>0$, where $\rho(i)$ is linear only to focus on nonlinearities coming from endogenous behavior. Then

$$
\begin{aligned}
r V_{0}= & n_{p} \theta \Delta_{1} \\
& \cdots \\
r V_{i}= & i \rho_{m}+n_{c} \tau_{i} \theta_{m c}\left(u-\Delta_{i}\right)+n_{p} \theta_{m p} \Delta_{i+1}-\delta \Delta_{i} \\
& \cdots \\
r V_{I}= & I \rho_{m}+n_{c} \tau_{I} \theta_{m c}\left(u-\Delta_{I}\right)-\delta \Delta_{I, I-1} .
\end{aligned}
$$

Thus $M$ with $i>0$ gets a flow $i \rho_{m}$, gets a share $\theta_{m c}$ of the surplus when he trades with $C$, suffers from depreciation at rate $\delta$, and increases $i$ when he meets $P$ as

\footnotetext{
${ }^{15}$ The process for $i$ is reminiscent of the one for currency holdings in monetary models with $m \in\{0,1,2, \ldots M\}$, like Green and Zhou (1998), Camera and Corbae (1999) or Berentsen (2002). However, those papers need to justify agents' decisions to trade 1 unit of money at a time, while here trading 1 unit of $x$ is guaranteed by technology and preferences.
} 
long as $i<I$. Similarly, we have

$$
r V_{p}=\rho_{p}+n_{c} \theta_{p c} u+\sum_{i=0}^{I-1} n_{i} \theta_{p m} \Delta_{i+1}
$$

Note that equilibrium is generically unique when $n_{p}$ is exogenous, because while $\boldsymbol{\tau}$ affects $\mathbf{n}$ it does not affect (23). Also, as in the baseline model, multiplicity obviously requires $\rho_{m}>0$. It is not hard to solve the model numerically and look for multiplicity with $n_{p}$ endogenous and $\rho_{m}>0$. First note that $V_{i}$ is nonlinear in $i$, and to see why this matters, consider a special case that we call reservation equilibria, defined as follows: for some $i^{*} \in\{1,2, \ldots I\}, \tau_{i}=1$ iff $i \geq i^{*}$. One might think all equilibria have this property, which would be true if $V_{i}$ were linear, but $V_{i}$ is not linear. Fig. 8, drawn for $I=5$, shows $\Delta_{i}=V_{i}-V_{i-1}$, and note that when $\Delta_{i}$ is increasing (decreasing) $V_{i}$ is convex (concave); hence $V_{i}$ starts convex and becomes concave as $i$ increases. ${ }^{16}$ In the left panel, with $\rho_{m}=0.05, \boldsymbol{\tau}=(1,0,1,1,1)$ is an equilibrium, and in fact the unique equilibrium for these parameters, but it is not a reservation equilibria ( $M$ trades when $i=1$ but not when $i=2)$. In the right panel, with $\rho_{m}=0.07, \boldsymbol{\tau}=(0,0,1,1,1)$ is a reservation equilibrium. Also shown are histograms for $\mathbf{n}$ and the value of $n_{p}$.
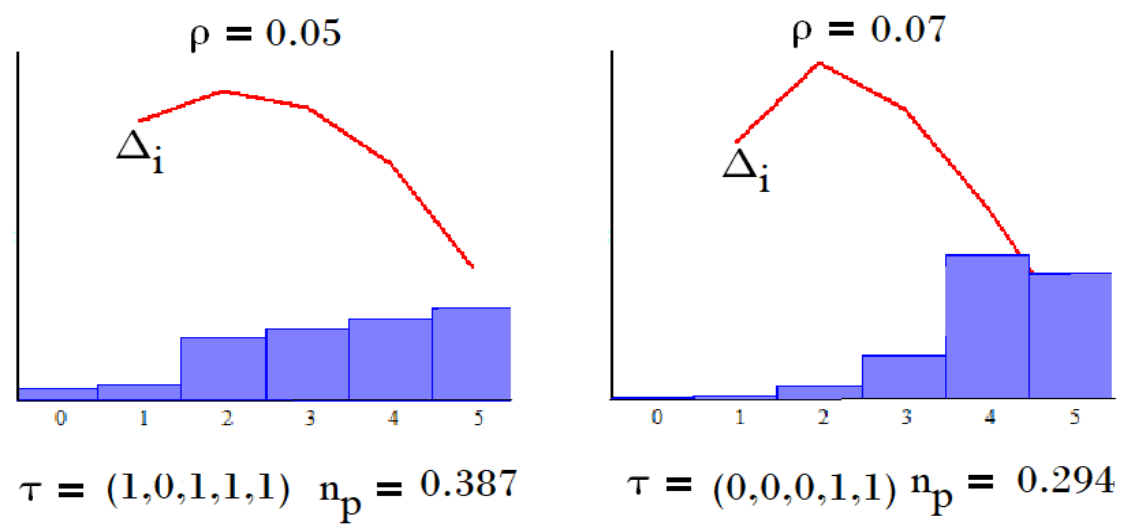

Figure 8: Equilibria at $\rho_{m}=0.05$ and $\rho_{m}=0.07$

\footnotetext{
${ }^{16}$ Parameters for this example, and for those below, unless indicated otherwise, are $u=1$, $r=0.01, \delta=0.09, n_{c}=0.25, \theta_{m p}=0.8, \theta_{p c}=\theta_{m c}=1, \rho_{p}=0$ and $\rho_{m}=0.05$.
} 
It is not hard to get multiplicity. The left panel of Figure 9 shows the result of checking all $2^{I}$ candidate equilibria for $I=100$ for each point on a grid in $\left(\rho_{m}, \delta\right)$ space. $^{17}$ The blue region has one equilibrium, the green has two, and yellow three. In this case they all happen to be reservation equilibria, implying multiplicity even within the special class, but one can also find reservation and other equilibria coexisting. The right panel checks for only reservation equilibrium when $I=50$. For every $i^{*} \in\{1,2, \ldots 50\}$ on the horizontal axis, the values of $\rho_{m}$ that support a reservation equilibrium with that $i^{*}$ are shown by bars. For low $\rho_{m}$ there is a reservation equilibrium with $i^{*}=0$, so $M$ trades with $C$ whenever he can. For high $\rho_{m}$ there is a reservation equilibrium with $i^{*}$ close to $I$, so $M$ rarely trades with $C$. Note that the overlap of the bars again demonstrates multiplicity. Also, note that for intermediate $i^{*}$ no value of $\rho_{m}$ supports reservation equilibria, and so reservation equilibria are not pervasive.
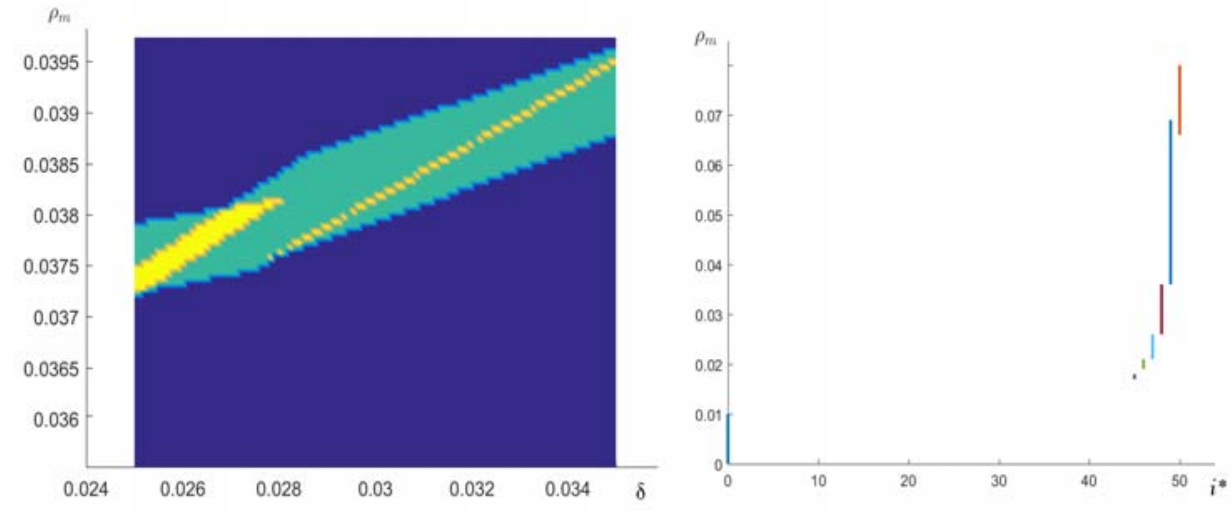

Figure 9: All equilibria with $I=100$ and reservation equilibria with $I=50$

The intuition is similar to $I=1$. First, one can show higher $\tau_{i}$ lowers $n_{i}$ and increases $n_{j}$ for $j \neq i$. Also, setting $\tau_{i}=\tau \forall i$ and increasing $\tau$ twists the inventory distribution - i.e., there is an $i_{\tau}$ such that $n_{i}$ decreases for $i>i_{\tau}$ and increases for $i \leq i_{\tau}$. So when $M$ is more inclined to trade with $C$, in the sense that $\tau_{i}=1$ for more values of $i$, a random middleman is more likely to have lower

\footnotetext{
${ }^{17}$ The parameter values are the same as above except $\delta=0.11$ in the left panel and $\delta=0.10$ in the right panel, mainly to make the pictures look nicer.
} 
inventory. This increases profit for $P$, and to re-establish $V_{p}=V_{0}$ we need to raise $n_{p}$, which encourages $M$ to trade with $C$ more often. This strategic effect makes multiplicity possible even when $I$ is big, and shows that insights from our tractable benchmark model go through for $I>1$.

\section{Conclusion}

This project continued the development of search-based theories of intermediation, with emphasis on endogenizing the measures of middlemen and producers, $n_{m}$ and $n_{p}$. The analysis delivered clean results on existence, efficiency and dynamics, with some predictions that were surprising, like the possibility of $\partial n_{m} / \partial \gamma_{m}>0$. A result we find interesting is this: absent devices like increasing returns, in models where $n_{m}$ and $n_{p}$ are endogenous, equilibrium is unique and simple if holding inventories involves storage costs, as in goods markets; but multiplicity and complicated dynamics emerge if holding inventories involves positive returns, as in asset markets, if we endogenize the composition of the market. There are many extensions and applications one can imagine for future work, but we think the existing findings already taught us a lot about search theory and about intermediation. 


\section{References}

[1] G. Afonso and R. Lagos (2015) "Trade Dynamics in the Market for Federal Funds," Econometrica 83, 263-313.

[2] G. Akerlof and R. Shiller (2009) Animal Spirits: How Human Psychology Drives the Economy, and Why It Matters for Global Capitalism. Princeton.

[3] S. Aruoba, G. Rocheteau and C. Waller (2007) "Bargaining and the Value of Money," JME 54, 2636-55.

[4] A. Berentsen (2002) "On the Distribution of Money Holdings in a RandomMatching Model," International Economic Review 43, 945-54.

[5] G. Biglaiser (1993) "Middlemen as Experts," RAND 24, 212-223.

[6] M. Boldrin, N. Kiyotaki and R. Wright (1993) "A Dynamic Equilibrium Model of Search, Production and Exchange," JEDC 17, 723-58.

[7] K. Burdett and M. Coles (1997) "Marriage and Class," QJE 112, 141-68.

[8] G. Camera (2001) "Search, Dealers, and the Terms of Trade," RED 4, 680694.

[9] G. Camera and D. Corbae (1999) "Money and Price Dispersion," IER 40, 985-1008.

[10] R. Cavalcanti and N. Wallace (1999) "A Model of Private Banknote Issue," RED 2, 104-36.

[11] M. Coles and R. Wright (1998) "A Dynamic Model of Search, Bargaining, and Money," JET 78, 32-54.

[12] P. Diamond (1982) "Aggregate Demand Management in Search Equilibrium," JPE 90, 881-94.

[13] P. Diamond and D. Fudenberg (1989) "Rational Expectations Business Cycles in Search Equilibrium," JPE 97, 606-19.

[14] D. Duffie, N. Gârleanu and L. Pederson (2005) "Over-the-Counter Markets," Econometrica 73, 1815-47.

[15] M. Farboodi, G. Jarosch and G. Menzio (2017) "Tough Middlemen," mimeo. 
[16] M. Farboodi, G. Jarosch and R. Shimer (2018) "Meeting Technologies in Decentralized Asset Markets," mimeo.

[17] M. Friedman (1960) A Program for Monetary Stability, Fordham University Press, New York.

[18] G. Gong (2019) "Middlemen in Search Models with Intensive and Extensive Margins," mimeo.

[19] E. Green and R. Zhou (1998) "A Rudimentary Random-Matching Model with Divisible Money and Prices," Journal of Economic Theory 81, 252-71.

[20] C. Gu, F. Mattesini, C. Monnet and R. Wright (2013) "Endogenous Credit Cycles," JPE 121, 940-65.

[21] A. Hosios (1990) "On the Efficiency of Matching and Related Models of Search and Unemployment," RES 57, 279-298.

[22] J. Hugonnier, B. Lester and P. Weill (2019) "Frictional Intermediation in Over-the-Counter Markets," FRB Philadelphia Working Paper 19-10.

[23] A. Johri and J. Leach (2002) "Middlemen and the Allocation of Heterogeneous Goods," IER 43, 347-361.

[24] E. Kalai (1977) "Proportional Solutions to Bargaining Situations: Interpersonal Utility Comparisons," Econometrica 45, 1623-30.

[25] G. Kaplan and G. Menzio (2016) "Shopping Externalities and Self-Fulfilling Unemployment Fluctuations," JPE 124, 771-825.

[26] N. Kiyotaki and R. Wright (1993) "A Search-Theoretic Approach to Monetary Economics," AER 83, 63-77.

[27] Y. Li (1998) "Middlemen and Private Information," JME 42, 131-159.

[28] R. Lagos, G. Rocheteau and R. Wright (2017) "Liquidity: A New Monetarist Perspective," JEL, in press.

[29] A. Masters (2007) "Middlemen in Search Equilibrium," IER 48, 343-62.

[30] A. Masters (2008) "Unpleasant Middlemen," JEBO 68, 73-86.

[31] D. Mortensen (1982) "Property Rights and Efficiency of Mating, Racing, and Related Games," AER 72, 968-79. 
[32] D. Mortensen (1999) "Equilibrium Unemployment Dynamics," IER 40, 889914.

[33] E. Nosal, Y.-Y. Wong and R. Wright (2015) "More on Middlemen: Equilibrium Entry and Efficiency in Intermediated Markets," JMCB 47, 7-37.

[34] C. Pissarides (2000) Equilibrium Unemployment Theory. MIT Press.

[35] C. Reinhart and K. Rogoff (2009) This Time Is Different: Eight Centuries of Financial Folly. Princeton University Press.

[36] G. Rocheteau and E. Nosal (2017) Money, Payments, and Liquidity. MIT Press.

[37] G. Rocheteau and R. Wright (2013) "Liquidity and Asset Market Dynamics," JME 60, 275-94.

[38] A. Rolnick and W. Weber (1986) "Inherent Instability in Banking: The Free Banking Experience," Cato Journal 5, 877-890.

[39] A. Rubinstein and A. Wolinsky (1987) "Middlemen," QJE 102, 581-594.

[40] A. Shevchenko (2004) "Middlemen," IER 45, 1-24.

[41] E. Smith (2004) "Intermediated Search," Economica 71, 619-636.

[42] A. Trejos and R. Wright (2016) "Search-Based Models of Money and Finance: An Integrated Approach," JET 164, 10-31.

[43] C. Tse (2009) "The Spatial Origin of Commerce," mimeo.

[44] X. Vives (2016) Competition and Stability in Banking: The Role of Regulation and Competition Policy. Princeton.

[45] M. Watanabe (2010) "A Model of Merchants," JET 145, 1865-1889.

[46] R. Wright (1995) "Search, Evolution and Money," JEDC 19, 181-206.

[47] R. Wright and Y.-Y. Wong (2014) "Buyers, Sellers and Middlemen: Variations on Search-Theoretic Themes," IER 55, 375-398. 


\section{Appendix A: Payment Frictions and Dynamics}

To begin let us suppose $U^{\prime \prime}(y)<0$. This is interesting because now we can have $U(y)<y$, implying the cost to the payer exceeds the value to the payee, which discourages intermediation because it requires two payments, $M$ to $P$ and $C$ to $M$, rather than one, $C$ to $P$. Thus we can interpret the model in terms of payment frictions. Given that, we can show uniqueness in goods markets also holds with nonlinear utility. For tractability, let us use Kalai's (1977) "proportional" bargaining solution, which has several advantages in related models (Aruoba et al. 2007).

To reduce notation, set $\theta_{p c}=\theta_{m c}=1$, which is fairly innocuous since type $C$ really plays a very minro role anyway, and convenient because it implies $V_{c}=0$ and $y_{c p}=y_{c m}=u$. Also, let $z \equiv U(u)$ and write the dynamic programming equations as

$$
\begin{aligned}
& r V_{p}=n_{c} z+n_{0} U\left(y_{m p}\right)-\gamma_{p}+\dot{V}_{p} \\
& r V_{0}=\left(1-n_{c}-n_{1}-n_{0}\right) \frac{1-\theta_{p m}}{\theta_{p m}} U\left(y_{m p}\right)+\dot{V}_{0} \\
& r V_{1}=n_{c}(z-\Delta)-\gamma_{m}+\dot{V}_{1} .
\end{aligned}
$$

The definition of equilibrium is similar, but the analysis is harder. To begin, let us first analyze steady state, then take up dynamics. Here are generalizations of some basic results for $U(y)=y$ :

Lemma $8 A$ (subgame perfect) class 0 equilibrium exists iff $\gamma_{p} \geq n_{c} z$ and $\gamma_{p} \geq$ $G\left(\gamma_{m}\right)$, where

$$
G\left(\gamma_{m}\right) \equiv n z+U\left(y_{0}\right)(1-n)
$$

and $y_{0}$ is given by the bargaining solution for $y_{m p}$ at $\mu=0$.

Lemma 9 A class 1 equilibrium exists iff $\gamma_{p} \leq n_{c} z$ and $\gamma_{p} \leq F\left(\gamma_{m}\right)$, where

$$
F\left(\gamma_{m}\right) \equiv n_{c} z-U(\bar{y})\left(1-n_{c}\right) \frac{\left(1-\theta_{p m}\right)}{\theta_{p m}},
$$

and $\bar{y}$ is the bargaining solution for $y_{m p}$ at $\mu=\bar{\mu}$.

Lemma 10 A class 2 equilibrium exists iff $\tilde{Q}\left(0, y_{0}\right)>0>\tilde{Q}(\bar{\mu}, \bar{y})$, where $\tilde{Q}$ is defined in the proof. 

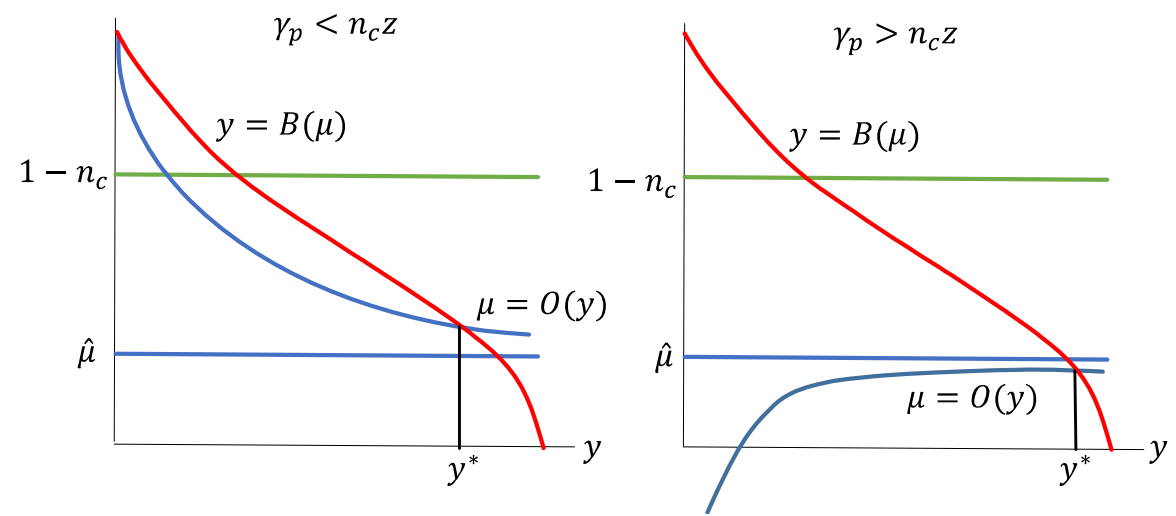

Figure 10: Equilibrium in $\left(y_{m p}, \mu\right)$ space

The main complication comes from the fact that, with a general $U(y)$, we cannot eliminate $y_{m p}$ from the above conditions. Hence, we work with two curves in $\left(y_{m p}, \mu\right)$ space, one representing bargaining and one representing the choice to be type $M$ or $P$. Setting $V_{0}=V_{p}$ implies a quadratic that solves for

$$
\mu=\frac{\left[2 \theta_{p m}\left(1-n_{c}\right)+n_{c}\right] U\left(y_{m p}\right)+\theta_{p m}\left(n_{c} z-\gamma_{p}\right)-\sqrt{\tilde{D}}}{2 \theta_{p m} U\left(y_{m p}\right)}
$$

where $\tilde{D}$ is the discriminant. This defines a function $\mu=O\left(y_{m p}\right)$, with $O$ for "occupational choice." One can check $\partial O / \partial y_{m p} \simeq-\left(n_{c} z-\gamma_{p}\right)$, where $a \simeq b$ means $a$ and $b$ have the same sign. As shown in Fig. 10, this traces a curve in $\left(y_{m p}, \mu\right)$ space that slopes up or down, depending on the sign of $n_{c} z-\gamma_{p}$, but in any case $\lim _{y_{m p} \rightarrow \infty} O\left(y_{m p}\right)=\hat{\mu} \in(0, \bar{\mu})$.

Next, use (??)-(??) to solve for $V_{1}-V_{0}$ and eliminate it from the bargaining solution to get $y_{m p}=B(\mu)$, where $B$ is for "bargaining." The result is

$$
\mu=B^{-1}\left(y_{m}\right)=\frac{\theta_{p m}\left(n_{c} z-\gamma_{m}\right)-\Upsilon}{\theta_{p m}\left(n_{c} z-\gamma_{m}\right)-\Upsilon+n_{c}\left(1-\theta_{p m}\right) U\left(y_{m p}\right)},
$$

where $\Upsilon \equiv\left(r+n_{c}\right)\left[\theta_{p m} y_{m p}+\left(1-\theta_{p m}\right) U\left(y_{m p}\right)\right]$. This traces a downward-sloping curve. Now we have

Lemma $11 y_{m p}=B(\mu)$ and $\mu=O\left(y_{m p}\right)$ intersect in $(0, \infty) \times(0, \bar{\mu})$ iff $F\left(\gamma_{m}\right)<$ $\gamma_{p}<G\left(\gamma_{m}\right)$. They never intersect more than once in $(0, \infty) \times(0, \bar{\mu})$. In $\left(\gamma_{p}, \gamma_{m}\right)$ space, $F$ is increasing and concave, $G$ is decreasing and concave, and $F\left(n_{c} z\right)=$ 
$G\left(n_{c} z\right)=n_{c} z$

Proposition 6 With $\gamma_{j}>0$ and $U^{\prime \prime}<0$ equilibrium exists and is generically unique.

Based on this is it not hard to show the results look a lot like Fig. 1 except now the boundaries of the different regions are nonlinear. In any case, the point it that uniqueness with $\gamma_{j}>0$ extends holds to nonlinear $U(y)$.

Now consider going dynamics in class 2 equilibrium, still allowing $U^{\prime \prime}<0$ and setting $\theta_{p c}=\theta_{m c}=1$, so again $y_{c p}=y_{c m}=u, V_{c}=0$ and $z=U(u)$. Also, here it is more convenient to work with the $n$ 's, rather than $\mu$, so let the state variable be $n_{1}$, the measure of $M$ with $x$, while $n_{0}=n_{m}-n_{1}$. Then

$$
\dot{n}_{1}=n_{0}\left(1-n_{c}-n_{1}-n_{0}\right)-n_{1} n_{c} .
$$

The other state is $\Delta=V_{1}-V_{0}$ capturing agents beliefs about the value of acquiring $x$. The bargaining solution is

$$
U\left(y_{m p}\right)=\theta_{p m}\left[U\left(y_{m p}\right)-y_{m p}+\Delta\right]
$$

While this system appears comples, it can be reduced to something manageable as follows: First, notice $V_{p}=V_{0} \forall t$ implies $\dot{V}_{p}=\dot{V}_{0} \forall t$. Then the dynamic programming equations imply that for $n_{m} \in\left(0,1-n_{c}\right)$ we have

$$
n_{c} z+n_{0} U\left(y_{m p}\right)-\gamma_{p}-\left(1-n_{c}-n_{0}-n_{1}\right) \frac{1-\theta_{p m}}{\theta_{p m}} U\left(y_{m p}\right)=0
$$

They also imply

$$
r \Delta=n_{c}(z-\Delta)-\gamma_{m}+\dot{\Delta}-\left(1-n_{c}-n_{0}-n_{1}\right) \frac{1-\theta_{p m}}{\theta_{p m}} U\left(y_{m p}\right)=0
$$

Using (34) and simplifying this, we get

$$
\dot{\Delta}=\left(r+n_{c}\right) \Delta+\gamma_{m}-\gamma_{p}+n_{0} U\left(y_{m p}\right)
$$

Thus (32) and (35) deliver a two-dimensional dynamical system in $\left(n_{1}, \Delta\right)$, with $n_{0}$ and $y_{m p}$ implicit functions of the state. 

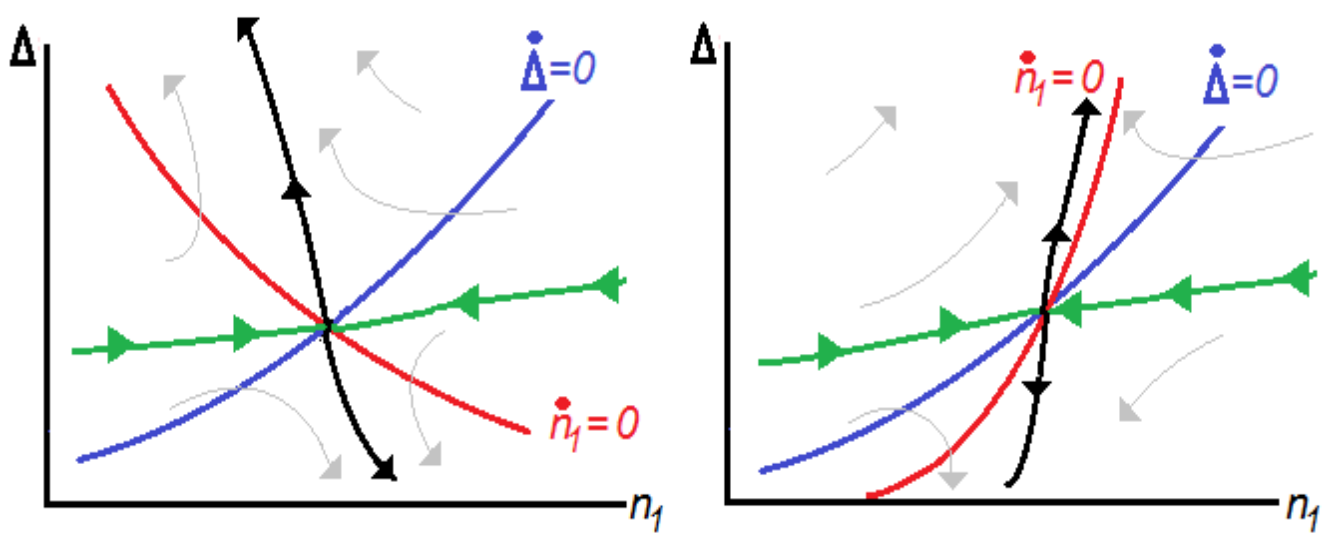

Figure 11: The phase plane with $\gamma_{j}>0$

As is standard, given an initial condition $\bar{n}_{1}$, an equilibrium is a nonnegative and bounded path for $\left(n_{1}, \Delta\right)$ solving (32) and (35) (boundedness comes from a transversality considerations discussed in, e.g., Rocheteau and Wright 2013). For $\gamma_{j}>0$ there is a unique steady state where the curves solving $\dot{n}_{1}=0$ and $\dot{\Delta}=0$. These have slopes

$$
\begin{aligned}
\left.\frac{\partial \Delta}{\partial n_{1}}\right|_{\dot{n}_{1}=0} & =\frac{\left[\left(n_{0}+n_{c}\right)+\left(1-n_{c}-n_{1}-2 n_{0}\right)\left(1-\theta_{p m}\right)\right]\left[\left(1-\theta_{p m}\right) U^{\prime}+\theta_{p m}\right]}{\left(1-n_{c}-n_{1}-2 n_{0}\right) \frac{U^{\prime}}{U}\left[\left(1-\theta_{p m}\right)\left(1-n_{c}-n_{1}\right)-n_{0}\right] \theta_{p m}} \\
\left.\frac{\partial \Delta}{\partial n_{1}}\right|_{\dot{\Delta}_{1}=0} & =\frac{U\left(1-\theta_{p m}\right)\left[\left(1-\theta_{p m}\right) U^{\prime}+\theta_{p m}\right]}{\left(r+n_{c}\right)\left[\left(1-\theta_{p m}\right) U^{\prime}+\theta_{p m}\right]+U^{\prime}\left(1-\theta_{p m}\right)\left(1-n_{c}-n_{1}\right) \theta_{p m}} .
\end{aligned}
$$

One can check the slope of $\dot{\Delta}=0$ is strictly positive, and while the slope of $\dot{n}_{1}=0$ can be positive or negative, when it is positive it is steeper than the $\dot{\Delta}=0$ curve. Also, $\partial \dot{n}_{1} / \partial n_{1}<0$ and $\partial \dot{\Delta} / \partial n_{1}<0$. Hence the phase portrait looks like Fig. 11, and whether $\dot{n}_{1}=0$ slopes up or down, the steady state is a saddle point: $\forall \bar{n}_{1}$ there is a unique $\bar{\Delta}$ such that starting at $\left(\bar{n}_{1}, \bar{\Delta}\right)$ the system $\left(n_{1}, \Delta\right)$ transits to steady state, and otherwise the system follows an explosive path. In other words, given any initial $n_{1}$ equilibrium and not only steady state is unique. 


\section{Appendix B: Proofs of Nonobvious Results}

Lemma 1: Class 0 and class 2 equilibria coexist in the region where $\gamma_{p} \geq$ $\bar{\gamma}_{p}$ and $\gamma_{m}<g\left(\gamma_{p}\right)$, but we claim the former is not subgame perfect. Notice $\gamma_{m}<\bar{\gamma}_{m}$ in this region, and consider a class 0 candidate equilibrium. Suppose a nonparticipant deviates and produces. When he meets another nonparticipant, which happens with positive probability, that agent has a strict incentive to accept his good and act like type $M$ because $\gamma_{m}<\bar{\gamma}_{m}$ (i.e., it is not credible to think he would reject it). This constitutes a profitable deviation.

Lemma 3: There are three ways for a convex $Q(\mu)=0$ to have solutions in $(0, \bar{\mu}):($ a) one root with $Q(0)<0<Q(\bar{\mu}) ;($ b) one root with $Q(0)>0>Q(\bar{\mu})$; (c) two-roots, which requires (c1) $Q(\bar{\mu})>0,(\mathrm{c} 2) Q(0)>0,(\mathrm{c} 3) Q^{\prime}(\bar{\mu})>0,(\mathrm{c} 4)$ $Q^{\prime}(0)<0$, and $(\mathrm{c} 5) Q\left(\mu^{*}\right)<0$, where $Q^{\prime}\left(\mu^{*}\right)=0$. Notice that

$$
\begin{aligned}
Q(0) & =\left(1-n_{c}\right) \theta_{p m}\left(\bar{\gamma}_{m}-\gamma_{m}\right)+\left(r+n_{c} \theta_{m c}\right)\left(\bar{\gamma}_{p}-\gamma_{p}\right) \\
Q(\bar{\mu}) & =n_{c}\left[r+n_{c} \theta_{m c}+\left(1-n_{c}\right) \theta_{m p}\right]\left(\bar{\gamma}_{p}-\gamma_{p}\right)-n_{c}\left(1-n_{c}\right) \theta_{m p}\left(\bar{\gamma}_{m}-\gamma_{m}\right) .
\end{aligned}
$$

In case (a), it is easy to see $Q(0)<0$ iff $\gamma_{p}>\bar{\gamma}_{p}+\left(1-n_{c}\right) \theta_{p m}\left(\bar{\gamma}_{m}-\gamma_{m}\right) /\left(r+n_{c} \theta_{m c}\right)$, and $Q(\bar{\mu})>0$ iff $\gamma_{p}<\bar{\gamma}_{p}-\left(1-n_{c}\right) \theta_{m p}\left(\bar{\gamma}_{m}-\gamma_{m}\right) /\left[r+n_{c} \theta_{m c}+\left(1-n_{c}\right) \theta_{m p}\right]$. As these conditions are contradictory, case (a) cannot occur.

Turning to case (c), (c1) $\Rightarrow \gamma_{p}<\bar{\gamma}_{p}$ while $(\mathrm{c} 2) \Rightarrow \kappa_{3}>0 \Rightarrow \gamma_{m}<g\left(\gamma_{p}\right)$, which is redundant given (c1) and that equilibrium requires that $\gamma_{m} \leq \bar{\gamma}_{m}$. Also, (c3) and (c4) $\Rightarrow$

$$
\begin{aligned}
& \gamma_{m}>\phi\left(\gamma_{p}\right) \equiv \bar{\gamma}_{m}+\frac{r+n_{c} \theta_{m c}-n_{c} \theta_{m p}}{n_{c}}\left(\bar{\gamma}_{p}-\gamma_{p}\right) \\
& \gamma_{m}<\psi\left(\gamma_{p}\right) \equiv \bar{\gamma}_{m}+\frac{r+n_{c} \theta_{m c}-n_{c} \theta_{m p}}{2\left(1-n_{c}\right) \theta_{p m}+n_{c}}\left(\bar{\gamma}_{p}-\gamma_{p}\right) .
\end{aligned}
$$

Finally, (c5) is equivalent to $D>0$, where $D$ is the discriminant of $Q(\mu)$.

We now show $r+n_{c} \theta_{m c}-n_{c} \theta_{m p}<0$ is necessary for (c3) and (c4). Suppose that $r+n_{c} \theta_{m c}-n_{c} \theta_{m p}>0$. This implies $\phi^{\prime}\left(\gamma_{p}\right)<0$ and $\psi^{\prime}\left(\gamma_{p}\right)<0$, and both of the lines $\gamma_{m}=\phi\left(\gamma_{p}\right)$ and $\gamma_{m}=\psi\left(\gamma_{p}\right)$ go through $\left(\bar{\gamma}_{p}, \bar{\gamma}_{m}\right)$. Since equilibrium requires $\gamma_{m} \leq \bar{\gamma}_{m}$ and $\gamma_{p} \leq \bar{\gamma}_{p}$, condition (c3) is violated, i.e., as illustrated in the left panel of Fig. 12, the intersection of conditions (c3) and (c4) is the empty set when $\gamma_{p} \leq \bar{\gamma}_{p}$. Suppose now that $r+n_{c} \theta_{m c}-n_{c} \theta_{m p}<0$. It is easy to show 


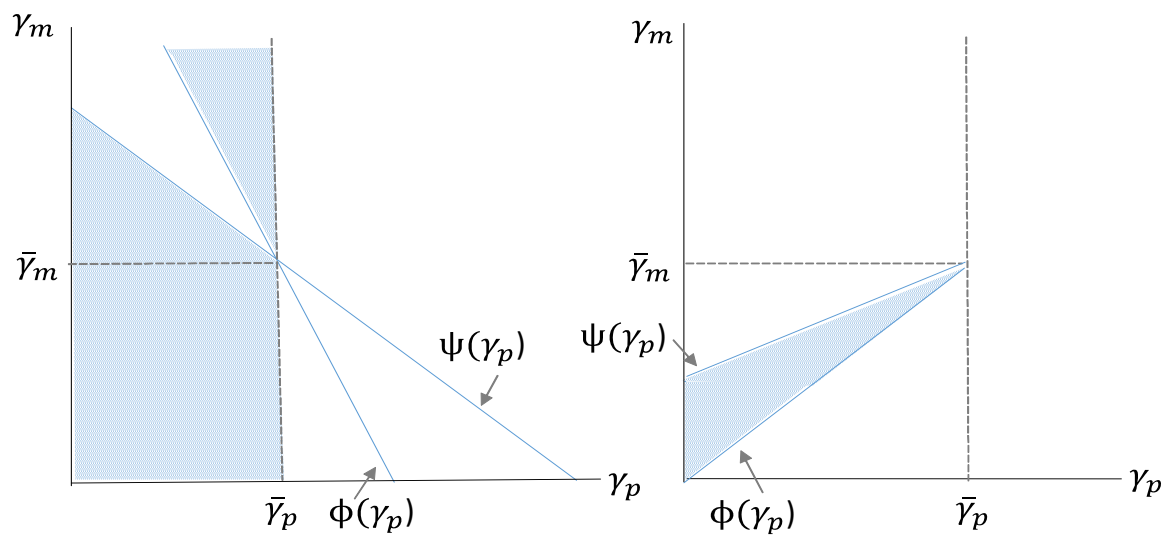

Figure 12: The functions $\phi\left(\gamma_{p}\right)$ and $\psi\left(\gamma_{p}\right)$

(c3) and (c4) are satisfied. The parameter set consistent with the conditions c(1), c(3) and c(4) is given by $\mathcal{S}_{1} \equiv\left\{\left(\gamma_{p}, \gamma_{m}\right) \mid 0<\gamma_{p} \leq \bar{\gamma}_{p}, \phi\left(\gamma_{p}\right)<\gamma_{m}<\psi\left(\gamma_{p}\right)\right\}$, shown in the right panel of Fig. 12

Similarly, let $\mathcal{S}_{2}$ be the set consistent with (c5). To characterize $\mathcal{S}_{2}$, the discriminant of $Q(\mu), D$, can itself be written as a quadratic in $\gamma_{m}$ given $\gamma_{p}$, $\hat{Q}\left(\gamma_{m} \mid \gamma_{p}\right)=\hat{\kappa}_{1} \gamma_{m}^{2}+\hat{\kappa}_{2} \gamma_{m}+\hat{\kappa}_{3}$, where

$$
\begin{aligned}
\hat{\kappa}_{1}= & n_{c}^{2}+4 n_{c}\left(1-n_{c}\right) \theta_{p m} \theta_{m p} \\
\hat{\kappa}_{2}= & -2 \bar{\gamma}_{m}\left[n_{c}{ }^{2}+4 n_{c}\left(1-n_{c}\right) \theta_{p m} \theta_{m p}\right] \\
& -2 n_{c}\left(\bar{\gamma}_{p}-\gamma_{p}\right)\left[\left(r+n_{c} \theta_{m c}-n_{c} \theta_{m p}\right)\left(1-2 \theta_{p m}\right)-2 \theta_{m p} \theta_{p m}\right] \\
\hat{\kappa}_{3}= & \bar{\gamma}_{m}^{2}\left[n_{c}{ }^{2}+4 n_{c}\left(1-n_{c}\right) \theta_{p m} \theta_{m p}\right]+\left(\bar{\gamma}_{p}-\gamma_{p}\right)^{2}\left(r+n_{c} \theta_{m c}-n_{c} \theta_{m p}\right) \\
& +2 n_{c} \bar{\gamma}_{m}\left(\bar{\gamma}_{p}-\gamma_{p}\right)\left[\left(r+n_{c} \theta_{m c}-n_{c} \theta_{m p}\right)\left(1-2 \theta_{p m}\right)-2 \theta_{m p} \theta_{p m}\right] .
\end{aligned}
$$

Since $\hat{\kappa}_{1}>0, \hat{Q}$ is strictly convex. Also, it is straightforward to show that $\hat{Q}\left(\bar{\gamma}_{m} \mid \gamma_{p}\right)<0 \forall \gamma_{p} \in\left[0, \bar{\gamma}_{p}\right)$. Thus, since $\hat{Q}$ is strictly convex and $\hat{Q}\left(\bar{\gamma}_{m} \mid \gamma_{p}\right)<0$, $\mathcal{S}_{2} \neq \varnothing \Rightarrow \hat{Q}\left(0 \mid \gamma_{p}\right)>0 \Rightarrow \hat{\kappa}_{3}>0$, as shown in the left panel of Fig. 13 .

It can be shown that $\hat{Q}\left(\gamma_{m} \mid \bar{\gamma}_{p}\right)>0 \forall \gamma_{m} \in\left[0, \bar{\gamma}_{m}\right)$ and $\hat{Q}\left(\bar{\gamma}_{m} \mid \bar{\gamma}_{p}\right)=0$. Since $\hat{Q}$ is continuous, $\hat{Q}\left(\gamma_{m} \mid \gamma_{p}\right)>0$ for some $\gamma_{m}<\bar{\gamma}_{m}$ if $\bar{\gamma}_{p}-\gamma_{p}$ is small. The admissible set of $\gamma_{p}$ for which $\hat{Q}\left(\gamma_{m} \mid \gamma_{p}\right)>0$ is pinned down by the lower root of $\hat{Q}\left(\gamma_{m} \mid \gamma_{p}\right)=0$ being positive, $\gamma_{m}^{-}\left(\gamma_{p}\right)=\left(-\hat{\kappa}_{2}-\sqrt{\Lambda}\right) / 2 \hat{\kappa}_{1}>0$, where $\Lambda=\hat{\kappa}_{2}^{2}-4 \hat{\kappa}_{1} \hat{\kappa}_{3}>0$. One 

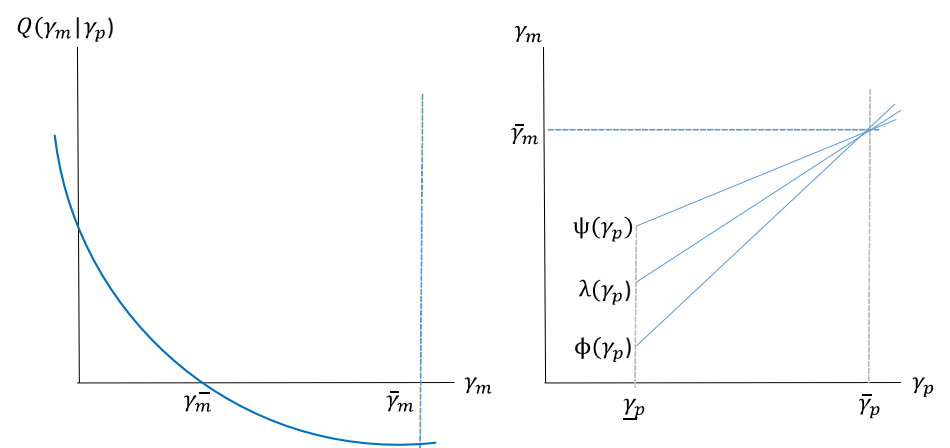

Figure 13: The functions $\hat{Q}\left(\gamma_{m} \mid \gamma_{p}\right)$ and $\lambda\left(\gamma_{p}\right)$

can show $\gamma_{m}^{-}\left(\gamma_{p}\right)>0 \Rightarrow \hat{\kappa}_{2}>0 \Rightarrow \gamma_{p}>\underline{\gamma}_{p}$ with

$\underline{\gamma}_{p} \equiv \bar{\gamma}_{p}+\bar{\gamma}_{m}\left[n_{c}+4\left(1-n_{c}\right) \theta_{p m} \theta_{m p}\right]\left[\left(r+n_{c} \theta_{m c}-n_{c} \theta_{m p}\right)\left(1-2 \theta_{p m}\right)-2 \theta_{m p} \theta_{p m}\right]$.

Given $\gamma_{p}$, the set of $\gamma_{m}$ such that $\hat{Q}\left(\gamma_{m} \mid \gamma_{p}\right)>0$ is $\left[0, \gamma_{m}^{-}\left(\gamma_{p}\right)\right)$. Therefore, $\mathcal{S}_{2}=$ $\left\{\left.\left(\gamma_{p}, \gamma_{m}\right)\right|_{p}<\gamma_{p}<\bar{\gamma}_{p}, 0<\gamma_{m}<\gamma_{m}^{-}\left(\gamma_{p}\right)\right\}$. Suppose for a given $\gamma_{p}$ there exists $\gamma_{m}^{-}\left(\gamma_{p}\right)>0$ such that $\hat{Q}\left(\gamma_{m}^{-}\left(\gamma_{p}\right)\right)=0$. Express the lower root as

$\gamma_{m}^{-}\left(\gamma_{p}\right)=\lambda\left(\gamma_{p}\right) \equiv \bar{\gamma}_{m}+\left(\bar{\gamma}_{p}-\gamma_{p}\right) \frac{n_{c}\left[\left(r+n_{c} \theta_{m c}-n_{c} \theta_{m p}\right)\left(1-2 \theta_{p m}\right)-2 \theta_{m p} \theta_{p m}\right]-\sqrt{\Lambda}}{n_{c}^{2}+4 n_{c}\left(1-n_{c}\right) \theta_{p m} \theta_{m p}}$

One can show $\lambda^{\prime}\left(\gamma_{p}\right)>0$. The right panel of Fig. 13 depicts $\gamma_{m}=\phi\left(\gamma_{p}\right), \gamma_{m}=$ $\psi\left(\gamma_{p}\right)$ and $\gamma_{m}=\lambda\left(\gamma_{p}\right)$. Since $\hat{Q} \equiv D>0 \Rightarrow \gamma_{m}<\lambda\left(\gamma_{h}\right)$, a necessary condition for case (c) is $\lambda^{\prime}\left(\gamma_{p}\right)<\phi^{\prime}\left(\gamma_{p}\right)$, as in the right panel of Fig. 13 .

Hence, (c) requires $\mathcal{S}_{1} \cap \mathcal{S}_{2} \neq \varnothing$ and $\lambda^{\prime}\left(\gamma_{p}\right)<\phi^{\prime}\left(\gamma_{p}\right)$. The inequality implies

$$
\begin{aligned}
\left(\theta_{m c}-\theta_{m p}\right)\left[n_{c}+4\left(1-n_{c}\right) \theta_{p m} \theta_{m p}\right] & <\left[n_{c}\left(\theta_{m c}-\theta_{m p}\right)\left(1-2 \theta_{p m}\right)-2 \theta_{m p} \theta_{p m}\right] \\
& -\left\{\left[n_{c}\left(\theta_{m c}-\theta_{m p}\right)\left(1-2 \theta_{p m}\right)-2 \theta_{m p} \theta_{p m}\right]^{2}\right. \\
& \left.-\left(\theta_{m c}-\theta_{m p}\right)\left[n_{c}+4\left(1-n_{c}\right) \theta_{p m} \theta_{m p}\right]\right\}^{1 / 2},
\end{aligned}
$$

ignoring terms with $r$ that strengthen the inequality. This implies

$$
-1+n_{c}\left(\theta_{m c}-\theta_{m p}\right)>4 n_{c} \theta_{m c} \theta_{p m}+4\left(1-n_{c}\right) \theta_{p m} \theta_{m p}\left(\theta_{m c}+\theta_{p m}\right) .
$$

But the LHS is negative and the RHS positive - a contradiction. 
Lemma 5: We derive

$$
\frac{\partial Q(\mu)}{\partial \gamma_{m}}=n_{c} \mu+\theta_{p m}\left[2\left(1-n_{c}\right) \mu-\left(1-n_{c}\right)-\mu^{2}\right] .
$$

One can check this vanishes when $\gamma_{p}=\bar{\gamma}_{p}$. Moreover,

$$
\left.\frac{\partial}{\partial \mu} \frac{\partial Q(\mu)}{\partial \gamma_{m}}\right|_{\mu=\tilde{\mu}}=n_{c}+\theta_{p m} 2\left(1-n_{c}\right)-2 \theta_{p m} \mu>0 .
$$

Hence, $\partial \mu / \partial \gamma_{m}>0$ if $\gamma_{p}<\bar{\gamma}_{p}$ and $\partial \mu / \partial \gamma_{m}<0$ if $\gamma_{p}>\bar{\gamma}_{p}$.

Proposition 2: There are two cases: First, if $\gamma_{m}>n_{c} u$ then $n_{m}>0$ cannot be efficient. In this case $\gamma_{p}>n_{c} u$ implies $n_{p}^{o}=0$ and $\gamma_{p}<n_{c} u$ implies $n_{p}^{o}=1-n_{c}$. Second, if $\gamma_{m}<n_{c} u$ then $n_{m}>0$ may or may not be efficient. Eliminating $n_{p}$ and $n_{m}$ in (10), we reduce the problem to

$$
\max _{\mu \in[0, \bar{\mu}]}\left\{\mu n_{c} u-n_{c} \frac{\mu}{1-\mu} \gamma_{p}-\mu \frac{1-n_{c}-\mu}{1-\mu} \gamma_{m}\right\} .
$$

The derivative of this is proportional to

$$
Q^{o}(\mu)=(1-\mu)^{2}\left(n_{c} u-\gamma_{m}\right)+n_{c}\left(\gamma_{m}-\gamma_{p}\right)
$$

This implies the solution is $\mu^{o}=0$ when $\gamma_{m} \geq g^{o}\left(\gamma_{p}\right)$, and $\mu^{o}=\bar{\mu}$ when $\gamma_{m} \geq$ $f^{o}\left(\gamma_{p}\right)$, with $f^{o}$ and $g^{o}$ defined in (11). If $\gamma_{m}<f^{o}\left(\gamma_{p}\right), g^{o}\left(\gamma_{p}\right)$ the solution is the unique $\mu^{o} \in(0, \bar{\mu})$ solving $Q^{o}\left(\mu^{o}\right)=0$.

Lemma 6: As $\partial\left(n_{m} \mu\right) / \partial \gamma_{m}=\partial\left(n_{m} \mu\right) / \partial \mu \times \partial \mu / \partial \gamma_{m}$ we need to sign $\partial\left(n_{m} \mu\right) / \partial \mu$. Notice $n_{m} \mu=\mu-n_{c} \mu /(1-\mu)$, which implies

$$
\frac{\partial\left(n_{m} \mu\right)}{\partial \mu} \simeq(1-\mu)^{2}-n_{c} \simeq \gamma_{p}-n_{c} u
$$

where $a \simeq b$ means $a$ and $b$ have the same sign. When $\gamma_{p}<n_{c} u, \partial \mu / \partial \gamma_{m}>0$ and $\partial\left(n_{m} \mu\right) / \partial \mu<0$, so $\partial\left(n_{m} \mu\right) / \partial \gamma_{m}<0$; when $\gamma_{p}>n_{c} u, \partial \mu / \partial \gamma_{m}<0$ and $\partial\left(n_{m} \mu\right) / \partial \mu>0$, so again $\partial\left(n_{m} \mu\right) / \partial \gamma_{m}<0$.

Proposition 3: First, it is obvious that the efficient and equilibrium outcomes correspond in general only if $\theta_{m c}=\theta_{p c}=1$, because that needed for $\bar{\gamma}_{p}=\bar{\gamma}_{m}=$ 
$n_{c} u$. With $\theta_{m c}=\theta_{p c}=1$, we have

$$
\begin{aligned}
f\left(\gamma_{p}\right) & =\frac{-n_{c}^{2} u+\left[1-\theta_{p m}\left(1-n_{c}\right)\right] \gamma_{p}}{\left(1-n_{c}\right)\left(1-\theta_{p m}\right)} \\
g\left(\gamma_{p}\right) & =\frac{n_{c} u\left[n_{c}+\theta_{p m}\left(1-n_{c}\right)\right]-n_{c} \gamma_{p}}{\left(1-n_{c}\right) \theta_{p m}} .
\end{aligned}
$$

If $\theta_{p m}^{o}=1$ then $g\left(\gamma_{p}\right)=g^{0}\left(\gamma_{p}\right)$; so for $\left(\gamma_{p}, \gamma_{m}\right) \in S_{0}^{o}, n_{j}=n_{j}^{o}$. If $\theta_{p m}^{o}=0$ then $f\left(\gamma_{p}\right)=f^{0}\left(\gamma_{p}\right)$; so for $\left(\gamma_{p}, \gamma_{m}\right) \in S_{1}^{o}$, again $n_{j}=n_{j}^{o}$. If $\theta_{p m}^{o} \in(0,1)$ then $\gamma_{m} \leq f\left(\gamma_{p}\right)$ implies $\gamma_{m} \leq f^{o}\left(\gamma_{p}\right)$ and $\gamma_{m} \leq g\left(\gamma_{p}\right)$ implies $\gamma_{m} \leq g^{o}\left(\gamma_{p}\right)$. Set $\theta_{m c}=\theta_{p c}=1$ and $\mu=\mu^{o}$ to get $\theta_{p m}^{o}$. It is easy to check $\theta_{p m}^{o} \in(0,1)$.

Lemma 10: We need $n_{c} z>\gamma_{m}, V_{p}=V_{0}$, and $y_{m p}$ is the bargaining solution. Now $V_{p}=V_{0}$ iff $0=\tilde{Q}\left(\mu, y_{m p}\right)=\tilde{\kappa}_{1} \mu^{2}+\tilde{\kappa}_{2} \mu+\tilde{\kappa}_{3}=0$ where

$$
\begin{aligned}
& \tilde{\kappa}_{1}=\theta_{p m} U\left(y_{m p}\right) \\
& \tilde{\kappa}_{2}=-\left[2 \theta_{p m}\left(1-n_{c}\right)+n_{c}\right] U\left(y_{m p}\right)-\theta_{p m}\left(n_{c} z-\gamma_{p}\right) \\
& \tilde{\kappa}_{3}=\theta_{p m}\left(n_{c} z-\gamma_{p}\right)+\theta_{p m}\left(1-n_{c}\right) U\left(y_{m p}\right) .
\end{aligned}
$$

There are again three cases for $\tilde{Q}\left(\mu, y_{m p}\right)=0$ : (a) one root with $\tilde{Q}\left(0, y_{0}\right)<$ $0<\tilde{Q}(\bar{\mu}, \bar{y})$; (b) one root with $\tilde{Q}\left(0, y_{0}\right)>0>\tilde{Q}(\bar{\mu}, \bar{y})$; and (c) two roots, requiring (c1) $\tilde{Q}\left(0, y_{0}\right)>0,(\mathrm{c} 2) \tilde{Q}(\bar{\mu}, \bar{y})>0,(\mathrm{c} 3) \partial \tilde{Q}\left(\mu, y_{m p} \mid \mu=\bar{\mu}\right) / \partial \mu>0,(\mathrm{c} 4)$ $\partial \tilde{Q}\left(\mu, y_{m p} \mid \mu=0\right) / \partial \mu<0$, and $(\mathrm{c} 5) \tilde{Q}\left(\mu^{*}, y_{m p}\right)<0$, where $\partial \tilde{Q}\left(\mu^{*}, y_{m p}\right) / \partial \mu=0$. As in Lemma 3, case (a) is impossible. Also notice

$$
\begin{aligned}
\tilde{Q}\left(0, y_{0}\right) & =\theta_{p m}\left[n_{c} z-\gamma_{p}+U\left(y_{0}\right)\left(1-n_{c}\right)\right] \\
\tilde{Q}(\bar{\mu}, \bar{y}) & =n_{c}\left[\theta_{p m}\left(n_{c} z-\gamma_{p}\right)-U(\bar{y})\left(1-n_{c}\right)\left(1-\theta_{p m}\right)\right] .
\end{aligned}
$$

In case (c), (c1) implies $\gamma_{p}<G\left(\gamma_{m}\right)$ and (c2) implies $\gamma_{p}<F\left(\gamma_{m}\right)$. From (c3) and $(\mathrm{c} 4)$, we have

$$
\frac{\partial \tilde{Q}\left(\mu, y_{m p}\right)}{\partial \mu}=2 \theta_{p m} \mu U\left(y_{m p}\right)-\left(n_{c} z-\gamma_{p}\right) \theta_{p m}-U\left(y_{m p}\right)\left[n_{c}+2\left(1-n_{c}\right) \theta_{p m}\right]
$$

We need this positive at $\mu=\bar{\mu}$, which means $\gamma_{p}>K \equiv n_{c} z-U(\bar{y}) n_{c} / \theta_{p m}$, and at $\mu=0$, which means $\gamma_{p}<n_{c} z+U\left(y_{0}\right)\left[n_{c}+2\left(1-n_{c}\right) \theta_{p m}\right] / \theta_{p m}$. Given $(\mathrm{c} 2)$, (c1) and (c4) are not binding. Also, (c2) and (c3) imply $\gamma_{p}$ is between $K$ and $F$, 
which holds iff $\theta_{p m}<\left(1-2 n_{c}\right) /\left(1-n_{c}\right)$. Assume this is true and consider (c5). To get $\mu^{*}$, solve $\partial Q / \partial \mu=0$ to get

$$
\begin{aligned}
\tilde{Q}\left(\mu^{*}, y_{m p}\right) \simeq & -\left(n_{c} z-\gamma_{p}\right)\left[\left(n_{c} z-\gamma_{p}\right) \theta_{p m}+2 n_{c} U\left(y_{m p}\right)\left(1-2 \theta_{p m}\right)\right] \\
& -U\left(y_{m p}\right)^{2} n_{c}\left[1+4 \theta_{p m}\left(1-\theta_{p m}\right)\left(1-n_{c}\right)\right]
\end{aligned}
$$

We need $\tilde{Q}\left(\mu^{*}, y_{m p}\right)<0$. With a small abuse of notation, let $\tilde{Q}\left(\mu^{*}, y_{m p}\right) \equiv$ $\tilde{Q}\left(\gamma_{p}\right)<0$ where

$$
\begin{aligned}
\tilde{Q}\left(\gamma_{p}\right)= & -\theta_{p m} \gamma_{p}^{2}+2 n_{c}\left[U\left(y_{m p}\right)\left(1-2 \theta_{p m}\right)+\theta_{p m} z\right] \gamma_{p}-n_{c}^{2} z^{2} \theta_{p m} \\
& -n_{c}^{2} z U\left(y_{m p}\right)\left(1-2 \theta_{p m}\right)-n_{c} U\left(y_{m p}\right)^{2}\left[1+4 \theta_{p m}\left(1-\theta_{p m}\right)\left(1-n_{c}\right)\right] .
\end{aligned}
$$

For (c5) we seek the set of $\gamma_{p}$ such that $\tilde{Q}\left(\gamma_{p}\right)<0$. There are three possibilities: (c5.1) one root with $\tilde{Q}(K)<0<\tilde{Q}(F) ;($ c5.2) one root with $\tilde{Q}(K)>0>$ $\tilde{Q}(F)$; (c5.3) two roots, which requires $\tilde{Q}(K)<0<\tilde{Q}(F), \tilde{Q}^{\prime}(K)>0>\tilde{Q}^{\prime}(F)$, and $\tilde{Q}\left(\gamma_{p}^{*}\right)<0$, where $\tilde{Q}^{\prime}\left(\gamma_{p}^{*}\right)=0$. Given $\gamma_{p}=K$ and $n_{c} z-\gamma_{p}=U(\bar{y}) n_{c} / \theta_{p m}$, $\tilde{Q}(K)=-U(\bar{y})^{2} \frac{n_{c}^{2}}{\theta_{p m}}\left[1+2\left(1-2 \theta_{p m}\right)\right]-U(\bar{y})^{2} n_{c}\left[1+4 \theta_{p m}\left(1-\theta_{p m}\right)\left(1-n_{c}\right)\right]<0$ Given $\gamma_{p}=F\left(\gamma_{m}\right)$ and $n_{c} z-\gamma_{p}=U(\bar{y})\left(1-\theta_{p m}\right)\left(1-n_{c}\right) / \theta_{p m}$, $\tilde{Q}(F) \simeq-U(\bar{y})^{2}\left\{\left(1-\theta_{p m}\right)\left(1-n_{c}\right)\left[1+n_{c}-\theta_{p m}\left(1+3 n_{c}\right)+4 n_{c} \theta_{p m}^{2}\right]+n_{c} \theta_{p m}\right\}<0$, for $\left(1-2 n_{c}\right) /\left(1-n_{c}\right)>\theta_{p m}>0$. This rules out (c5.1) and (c5.2). To check (c5.3), consider

$$
\tilde{Q}^{\prime}\left(\gamma_{p}\right)=-2 \theta_{p m} \gamma_{p}+2 n_{c}\left[U\left(y_{m p}\right)\left(1-2 \theta_{p m}\right)+\theta_{p m} z\right]
$$

Now $\tilde{Q}^{\prime}\left(\gamma_{p}\right)>0$ at $\gamma_{p}=K$, and $\tilde{Q}^{\prime}\left(\gamma_{p}\right)>0$ at $\gamma_{p}=F\left(\gamma_{m}\right)$. As $\tilde{Q}^{\prime}(F)>0$ violates $(\mathrm{c} 5.3)$, there is no $\gamma_{p}^{*}$ between $K$ and $F$ such that $\tilde{Q}\left(\gamma_{p}^{*}\right)<0$.

Lemma 11: We need $B$ and $O$ to cross in $(0, \infty) \times(0, \bar{\mu})$, plus $\gamma_{m}<n_{c} z$. For $\mu \in(0, \bar{\mu})$, we check $\tilde{Q}\left(0, y_{0}\right)>0>\tilde{Q}(\bar{\mu}, \bar{y})$, where $y_{0}=B(0)$ and $\bar{y}=B(\bar{\mu})$. Now $\tilde{Q}\left(0, y_{0}\right)>0$ iff $\gamma_{p}<G\left(\gamma_{m}\right)$. At $\gamma_{m}=n_{c} z$, bargaining implies $y_{0}=0$ and $\gamma_{p}<G\left(\gamma_{m}\right)$ becomes $\gamma_{p}<n_{c} z$. As we lower $\gamma_{m}, y_{0}$ rises, and we need $\gamma_{p}<G\left(\gamma_{m}\right)$. In $\left(\gamma_{p}, \gamma_{m}\right)$ space $G\left(\gamma_{m}\right)$ traces a downward sloping and concave 
curve (see below), and $\tilde{Q}\left(0, y_{0}\right)>0$ to the left of $\gamma_{p}=G\left(\gamma_{m}\right)$. Then $\tilde{Q}(\bar{\mu}, \bar{y})<0$ iff $\gamma_{p}>F\left(\gamma_{m}\right)$. At $\gamma_{m}=n_{c} z$, bargaining implies $\bar{y}=0$, and $\gamma_{p}>F\left(\gamma_{m}\right)$ becomes $\gamma_{p}>n_{c} z$. As we lower $\gamma_{m}, \bar{y}$ rises, and we need $\gamma_{p}>F\left(\gamma_{m}\right)$. In $\left(\gamma_{p}, \gamma_{m}\right)$ space, $F\left(\gamma_{m}\right)$ traces a upward sloping and concave curve (see below). Hence $\exists \mu \in(0, \bar{\mu})$ solving $\tilde{Q}\left(\mu, y_{m p}\right)=0$ iff $F\left(\gamma_{m}\right)<\gamma_{p}<G\left(\gamma_{m}\right)$. To check $y_{m p}>0$, note from Fig. ?? that $y_{m p}>\bar{y} \geq 0$. To check $V_{p}=V_{0} \geq 0$, by construction $V_{0} \geq 0$ if $\mu \geq 0$.

To show $B$ and $O$ cannot cross more than once, write

$$
\mu=\frac{A-\left(r+n_{c}\right)\left(1-\theta_{p m}\right) U\left(y_{m p}\right)}{A-r\left(1-\theta_{p m}\right) U\left(y_{m p}\right)} \equiv \mu^{*},
$$

with $A=\theta_{p m}\left(n_{c} z-\gamma_{m}\right)-\left(r+n_{c}\right) \theta_{p m} y_{m p}>0$, from the bargaining solution. Note $\mu>0 \Rightarrow A>\left(r+n_{c}\right)\left(1-\theta_{p m}\right) U\left(y_{m p}\right)$, and $\mu<\bar{\mu} \Rightarrow A<$ $(1+r)\left(1-\theta_{p m}\right) U\left(y_{m p}\right)$. Then

$$
\begin{aligned}
\frac{\partial O\left(y_{m p}\right)}{\partial y_{m p}} & =-\frac{U^{\prime} \theta_{p m}\left(n_{c} z-\gamma_{p}\right)}{U \sqrt{\tilde{D}}}(1-\mu) \\
\frac{\partial B^{-1}\left(y_{m p}\right)}{\partial y_{m p}} & =-\frac{n_{c}\left(1-\theta_{p m}\right)}{\left[A-r\left(1-\theta_{p m}\right) U\right]^{2}}\left[A U^{\prime}+\theta_{p m}\left(r+n_{c}\right) U\right]
\end{aligned}
$$

If $n_{c} z<\gamma_{p}$ equilibrium is obviously unique. If $n_{c} z>\gamma_{p}$, we claim $\partial O / \partial y_{m p}>$ $\partial B^{-1} / \partial y_{m p}$ when they cross. To verify this, insert $\mu=\mu^{*}$ to get

$$
\frac{\partial O\left(y_{m p}\right)}{\partial y_{m p}}=-\frac{U^{\prime} \theta_{p m}\left(n_{c} z-\gamma_{p}\right)}{\sqrt{\tilde{D}}} \frac{n\left(1-\theta_{p m}\right)}{A-r\left(1-\theta_{p m}\right) U}
$$

where $\tilde{D}$ is the discriminant of $\tilde{Q}$. Using (30) to replace $\sqrt{\tilde{D}}$ and $\mu=\mu^{*}$, we get

$$
\frac{\partial O\left(y_{m p}\right)}{\partial y_{m p}}=-\frac{U^{\prime} \theta_{p m}\left(n_{c} z-\gamma_{p}\right) n_{c}\left(1-\theta_{p m}\right)}{\left[A-r\left(1-\theta_{p m}\right) U\right] \Omega}
$$

where $\Omega \equiv\left[2 \theta_{p m}\left(1-n_{c}\right)+n_{c}\right] U+\theta_{p m}\left(n_{c} z-\gamma_{p}\right)-\frac{2 \theta_{p m} U\left[A-\left(r+n_{c}\right)\left(1-\theta_{p m}\right) U\right]}{A-r\left(1-\theta_{p m}\right) U}$.

Now $A=\theta\left(n_{c} z-\gamma_{m}\right)-\left(r+n_{c}\right) \theta y^{*}$ and $U=U\left(y^{*}\right)$ solves

$$
\begin{aligned}
& \theta U[A-(r+n)(1-\theta) U]^{2}+\theta[A-r(1-\theta) U]^{2}\left[n z-\gamma_{p}+(1-n) U\right] \\
= & {[A-(r+n)(1-\theta) U][A-r(1-\theta) U]\left\{[2 \theta(1-n)+n] U+\theta\left(n z-\gamma_{p}\right)\right\} }
\end{aligned}
$$


Routine algebra implies $\partial O(y) / \partial y-\partial B^{-1}(y) / \partial y$ is proportional to

$$
\begin{aligned}
& U \theta\left(n z-\gamma_{p}\right)[A-r(1-\theta) U]\left[U^{\prime} r(1-\theta)+(r+n) \theta\right] \\
& +\left[A U^{\prime}+\theta(r+n) U\right] U\{n[A-r(1-\theta) U]+2 \theta n[(1+r)(1-\theta) U-A]\}
\end{aligned}
$$

Since $(1+r)(1-\theta) U>A>r(1-\theta) U$, this is positive, establishing the result.

Finally, for the properties of $F$ and $G$, derive

$$
\begin{aligned}
G^{\prime}\left(\gamma_{m}\right) & =\frac{-\theta_{p m}\left(1-n_{c}\right) U^{\prime}\left(y_{0}\right)}{\left(r+n_{c}\right)\left[\theta_{p m}+\left(1-\theta_{p m}\right) U^{\prime}\left(y_{0}\right)\right]}<0 \\
G^{\prime \prime}\left(\gamma_{m}\right) & \simeq \frac{-\theta_{p m}^{2}\left(1-n_{c}\right) U^{\prime \prime}\left(y_{0}\right) y_{0}^{\prime}\left(\gamma_{m}\right)}{\left(r+n_{c}\right)\left[\theta_{p m}+\left(1-\theta_{p m}\right) U^{\prime}\left(y_{0}\right)\right]^{2}}<0 .
\end{aligned}
$$

Thus $G(\cdot)$ is decreasing and concave in $\left(\gamma_{m}, \gamma_{p}\right)$ space or $\left(\gamma_{p}, \gamma_{m}\right)$ space . Similarly, $F^{\prime}\left(\gamma_{m}\right)>0$ and $F^{\prime \prime}\left(\gamma_{m}\right)>0$. Thus $F(\cdot)$ is increasing and convex in $\left(\gamma_{m}, \gamma_{p}\right)$ space, or increasing and concave in $\left(\gamma_{p}, \gamma_{m}\right)$ space.

Lemma 7: For preliminaries, first solve equations (??)-(??) for

$$
\begin{aligned}
r V_{p} & =\frac{\left(r+\tau n_{c} \theta_{m c}+n_{p} \theta_{m p}\right)\left(\bar{\gamma}_{p}-\gamma_{p}\right)+n_{m}(1-\mu) \theta_{p m}\left(\tau \bar{\gamma}_{m}-\gamma_{m}\right)}{r+\tau n_{c} \theta_{m c}+n_{p} \theta_{m p}} \\
r V_{0} & =\frac{n_{p} \theta_{m p}\left(\tau \bar{\gamma}_{m}-\gamma_{m}\right)}{r+\tau n_{c} \theta_{m c}+n_{p} \theta_{m p}} \\
r V_{1} & =\frac{\left(r+n_{p} \theta_{m p}\right)\left(\tau \bar{\gamma}_{m}-\gamma_{m}\right)}{r+\tau n_{c} \theta_{m c}+n_{p} \theta_{m p}}
\end{aligned}
$$

and notice steady state implies

$$
n_{p}=\frac{n_{c} \tau \mu}{1-\mu} \text { and } n_{m}=\frac{\left(1-n_{c}\right)(1-\mu)-n_{c} \tau \mu}{1-\mu} .
$$

We now consider each candidate equilibrium.

Equilibrium $1^{K}$ : In a candidate equilibrium with $\tau=0$ and $n_{m}=0,(36)$ (38) reduce to

$$
\begin{aligned}
& r V_{p}=\bar{\gamma}_{p}-\gamma_{p} \\
& r V_{0}=\left(1-n_{c}\right) \theta_{m p}\left(V_{1}-V_{0}\right) \\
& r V_{1}=-\gamma_{m} .
\end{aligned}
$$


The best response condition for $\tau=0$ is $V_{1}^{D} \leq V_{1}$, where $V_{1}^{D}$ is the value to setting $\tau=1$ then reverting to the candidate equilibrium with $V_{0}$ given by (41):

$$
\left(r+n_{c} \theta_{m c}\right) V_{1}^{D}=\bar{\gamma}_{m}-\gamma_{m}-\frac{n_{c} \theta_{m c}\left(1-n_{c}\right) \theta_{m p}}{\left[r+\left(1-n_{c}\right) \theta_{m p}\right]} \frac{\gamma_{m}}{r}
$$

Simplifying, $\tau=0$ is a best response iff $\gamma_{m} \leq \widehat{\gamma}_{m}$ where $\widehat{\gamma}_{m}$ is defined in (12). Similarly, for $n_{m}=0, V_{p} \geq V_{0}$ iff $\gamma_{m} \geq \widehat{f}\left(\gamma_{p}\right)$ where $\widehat{f}\left(\gamma_{p}\right)$ is defined in (13). Hence, class $1^{K}$ equilibrium exists iff $\widehat{f}\left(\gamma_{p}\right) \leq \gamma_{m} \leq \widehat{\gamma}_{m}$.

Equilibrium $1^{T}$ : Consider next $\tau=1$ and $n_{m}=0$. The best response condition for $\tau=1$ is $\gamma_{m} \geq \widehat{\gamma}_{m}$. For $n_{m}=0, V_{p} \geq V_{0}$ iff $\gamma_{m} \geq f\left(\gamma_{p}\right)$, where $f\left(\gamma_{p}\right)$ is defined in (7). Hence, class $1^{T}$ equilibrium exists iff $\gamma_{m} \geq \max \left\{\widehat{\gamma}_{m}, f\left(\gamma_{p}\right)\right\}$.

Equilibrium $2^{K}$ : Consider $\tau=0$ and $n_{m} \in\left(0,1-n_{c}\right)$, where the dynamic programming equations are the same as (40)-(42). The best response condition for $\tau=0$ is $V_{1}^{D} \leq V_{1}$, where $V_{1}^{D}$ is the the value to setting $\tau=1$ and reverting to the candidate strategy with $V_{0}=V_{p}$. Algebra implies

$$
V_{1}^{D}=\frac{r\left(\bar{\gamma}_{m}-\gamma_{m}\right)+n_{c} \theta_{m c}\left(\bar{\gamma}_{p}-\gamma_{p}\right)}{r\left(r+n_{c} \theta_{m c}\right)} .
$$

It is easy to check $V_{1}^{D} \leq V_{1}$ iff $\gamma_{m} \leq k\left(\gamma_{p}\right)$ where $k\left(\gamma_{p}\right)$ is defined in (14). The condition for $n_{m} \in\left(0,1-n_{c}\right), V_{p}=V_{0}$, now implies

$$
n_{p}=\frac{r\left(\bar{\gamma}_{p}-\gamma_{p}\right)}{\theta_{m p}\left(\gamma_{m}+\bar{\gamma}_{p}-\gamma_{p}\right)}
$$

Now $n_{p}<1-n_{c}$ is the binding condition for $n_{p} \in\left(0,1-n_{c}\right)$, and that holds iff $\gamma_{m} \leq \widehat{f}\left(\gamma_{p}\right)$. So class $2^{K}$ equilibrium exists iff $\gamma_{m} \leq \min \left\{k\left(\gamma_{p}\right), \widehat{f}\left(\gamma_{p}\right)\right\}$.

Equilibrium $2^{R}$ : Consider next $\tau \in(0,1)$ and $n_{m} \in\left(0,1-n_{c}\right)$. Now $\tau \in(0,1)$ iff $r V_{1}=-\gamma_{m}$ iff $\gamma_{m}=-\left[r+\left(1-n_{c}-n_{m}\right) \theta_{m p}\right] u$. Solve this for

$$
n_{m}=\frac{\gamma_{m}+\left[r+\left(1-n_{c}\right) \theta_{m p}\right] u}{u \theta_{m p}}
$$

One can check $n_{m} \in\left(0,1-n_{c}\right)$ implies

$$
\tau=\frac{\left(\gamma_{m}+r u\right)\left(\gamma_{m}+r u+\bar{\gamma}_{p}-\gamma_{p}\right)}{n_{c} u\left[\gamma_{m}+r u+\theta_{m p}\left(\bar{\gamma}_{p}-\gamma_{p}\right)+\theta_{p m} \theta_{m p}\left(1-n_{c}\right) u\right]}
$$


We now obtain the set of parameters such that $\tau \in(0,1)$ and $n_{m} \in\left(0,1-n_{c}\right)$. To see when $\tau>0$, denote the denominator of $\tau$ by $D$. There are two possibilities, $D<0$ and $D>0$. The former can be shown to be inconsistent with $\tau>0$, so we are left with $D>0$, which holds iff

$$
\gamma_{m}>\Psi\left(\gamma_{p}\right) \equiv-r u-\theta_{m p}\left(\bar{\gamma}_{p}-\gamma_{p}\right)-\theta_{p m} \theta_{m p}\left(1-n_{c}\right) u
$$

Given this, $\tau>0$ if $\gamma_{m}<k\left(\gamma_{p}\right)$. So $\tau>0$ iff $\Psi\left(\gamma_{p}\right)<\gamma_{m}<k\left(\gamma_{p}\right)$.

Also, using $D>0$, algebra implies $\tau<1$ iff $Q_{1}\left(\gamma_{m}\right)<0$ where $Q_{1}\left(\gamma_{m}\right)=$ $a_{1} \gamma_{m}^{2}+b_{1} \gamma_{m}+c_{1}<0$, with $a_{1}=1$,

$$
\begin{aligned}
& b_{1}=\bar{\gamma}_{p}-\gamma_{p}+2 r u-n_{c} u \\
& c_{1}=r u\left(\bar{\gamma}_{p}-\gamma_{p}+r u\right)-n_{c} u\left[r u+\theta_{m p}\left(\bar{\gamma}_{p}-\gamma_{p}\right)+\theta_{p m} \theta_{m p}\left(1-n_{c}\right) u\right] .
\end{aligned}
$$

We cannot sign $b$ or $c$, but can show $Q_{1}\left(\gamma_{m}^{*}\right)<0$, where $\gamma_{m}^{*}$ solves $Q_{1}^{\prime}\left(\gamma_{m}^{*}\right)=0$. Hence, there are three possibilities for $Q_{1}\left(\gamma_{m}\right)<0$, all of which reduce to $\gamma_{m}>$ $\widehat{k}\left(\gamma_{p}\right)$ where $\widehat{k}\left(\gamma_{p}\right)$ is the lower root of $Q_{1}\left(\gamma_{m}\right)=0$ :

1. if $\gamma_{m}^{*}>0$ then $Q_{1}(0)<0$ and so $Q_{1}\left(\gamma_{m}\right)<0$ iff $\gamma_{m}>\widehat{k}\left(\gamma_{p}\right)$;

2. if $\gamma_{m}^{*}<0$ and $Q_{1}(0)<0$ then $Q_{1}\left(\gamma_{m}\right)<0$ also implies $\gamma_{m}>\widehat{k}\left(\gamma_{p}\right)$;

3. if $\gamma_{m}^{*}<0$ and $Q_{1}(0)>0$ then $Q_{1}\left(\gamma_{m}\right)<0$ implies $\gamma_{m}^{+}>\gamma_{m}>\widehat{k}\left(\gamma_{p}\right)$,

where $\gamma_{m}^{+}$is the upper root of $Q_{1}\left(\gamma_{m}\right)=0$, but $\tau>0$ implies $\gamma_{m}^{+}>\gamma_{m}$ is not binding. All these possibilities imply, given the other conditions, that $\tau<1$ iff $Q_{1}\left(\gamma_{m}\right)<0$ iff $\gamma_{m}>\widehat{k}\left(\gamma_{p}\right)$.

We claim that when this holds, the earlier condition $\gamma_{m} \geq \Psi\left(\gamma_{p}\right)$ is not binding. To see this, notice $\widehat{k}\left(\gamma_{p}\right)$ intersects $\Psi$ at $\left(\widetilde{\gamma}_{p},-r u-\left(1-n_{c}\right) \theta_{m p} u\right)$ and $\left(\bar{\gamma}_{p}+\left(1-n_{c}\right) \theta_{p m} u,-r u\right)$, and $\widehat{k}\left(\gamma_{p}\right)$ is increasing and concave. Thus $\widehat{k}\left(\gamma_{p}\right)>$ $\Psi\left(\gamma_{p}\right)$, so the binding constraint is $\gamma_{m} \geq \widehat{k}\left(\gamma_{p}\right)$. In sum, class $2^{R}$ equilibrium exists iff $\widehat{k}\left(\gamma_{p}\right) \leq \gamma_{m} \leq k\left(\gamma_{p}\right)$.

Equilibrium $2^{T}$ : Consider $\tau=1$ and $n_{m} \in\left(0,1-n_{c}\right)$. We first solve $V_{p}=V_{0}$ for $\mu$ and check $\mu \in(0, \bar{\mu})$, since that is equivalent to $n_{m} \in\left(0,1-n_{c}\right)$, where $\bar{\mu}=1-n_{c}$. By (36)-(37), $V_{p}=V_{0}$ iff

$$
\left(r+n_{c} \theta_{m c}+n_{p} \theta_{m p}\right)\left(\bar{\gamma}_{p}-\gamma_{p}\right)+\left[n_{m} \theta_{p m}(1-\mu)-n_{p} \theta_{m p}\right]\left(\bar{\gamma}_{m}-\gamma_{m}\right)=0 .
$$


Using (39) to eliminate $n_{p}$ and $n_{m}$, then simplifying, we get $Q_{2}(\mu)=a_{2} \mu^{2}+b_{2} \mu+$ $c_{2}=0$ where

$$
\begin{aligned}
a_{2} & =\theta_{p m}\left(\bar{\gamma}_{m}-\gamma_{m}\right) \\
b_{2} & =-\left(r+n_{c} \theta_{m c}-n_{c} \theta_{m p}\right)\left(\bar{\gamma}_{p}-\gamma_{p}\right)-\left[n_{c}+2\left(1-n_{c}\right) \theta_{p m}\right]\left(\bar{\gamma}_{m}-\gamma_{m}\right) \\
c_{2} & =\left(r+n_{c} \theta_{m c}\right)\left(\bar{\gamma}_{p}-\gamma_{p}\right)+\left(1-n_{c}\right) \theta_{p m}\left(\bar{\gamma}_{m}-\gamma_{m}\right) .
\end{aligned}
$$

We need to check when the solution $Q_{2}(\mu)=0$ is in $(0, \bar{\mu})$. Now $Q_{2}(\mu)$ can have one or two roots. Since $Q_{2}(0)>0$ and $\mu>0$, the one root case corresponds to the lower-root of $Q_{2}(\mu)$, say $\mu^{-}$. To analyze $\mu^{-}$, first, notice $\mu^{-}>0$ requires $\gamma_{m}>\bar{\gamma}_{m}+\left(r+n_{c} \theta_{m c}\right)\left(\bar{\gamma}_{p}-\gamma_{p}\right) /\left[\left(1-n_{c}\right) \theta_{p m}\right]$, which is non-binding. Second, $\mu^{-}<1-n_{c}$ requires $\gamma_{m}<f\left(\gamma_{p}\right)$, which holds iff $Q_{2}(\bar{\mu})<0$. This last result implies a two-root result is impossible because we have $Q_{2}(0)>0$ and $Q_{2}(\bar{\mu})<0$. Hence only one root of $Q_{2}(\mu)=0$ can occur.

Finally, we check the best response condition for $\tau=1$, which reduces to $\gamma_{m} \geq-\left(r+n_{p} \theta_{m p}\right) u$, or

$$
\mu \leq \frac{\gamma_{m}+r u}{\gamma_{m}+r u-n_{c} \theta_{m p} u}
$$

Substituting $\mu$ using $\mu^{-}$and solving for $\gamma_{m}$ yields $Q_{1}\left(\gamma_{m}\right)=a_{1} \gamma_{m}^{2}+b_{1} \gamma_{m}+c_{1} \leq 0$, as in class $2^{R}$ equilibrium. So the set of $\gamma_{m}$ consistent with $Q_{1}\left(\gamma_{m}\right) \leq 0$ is $\gamma_{m} \geq \widehat{k}\left(\gamma_{p}\right)$. Hence, class $2 T$ equilibrium exists iff $\widehat{k}\left(\gamma_{p}\right) \leq \gamma_{m} \leq f\left(\gamma_{p}\right)$.

Proposition 4: Part (i) of the result follows directly from Lemma 7. For part (ii), for fixed $n_{p}$ and $n_{m}$ we have

$$
\begin{aligned}
r V_{p} & =n_{c} \theta_{p c} u+n_{m} \theta_{p m}(1-\mu)\left(V_{1}-V_{0}\right)-\gamma_{m} \\
r V_{1} & =n_{c} \theta_{m c} \tau\left(u+V_{0}-V_{1}\right)-\gamma_{m} \\
r V_{0} & =n_{c} \theta_{m p}\left(V_{1}-V_{0}\right)
\end{aligned}
$$

Consider a candidate equilibrium with $\tau=0$. Then $\mu=1$ and the above equations imply

$$
V_{0}=\frac{-n_{c} \theta_{m p} \gamma_{m}}{r\left(r+n_{c} \theta_{n p}\right)} \text { and } V_{1}=\frac{\gamma_{m}}{r}
$$


A deviation by $M$ to $\tau=1$ implies $r V_{1}^{d}=n_{c} \theta_{m c}\left(u+V_{0}-V_{1}^{d}\right)-\gamma_{m}$. After inserting $V_{0}$ we get

$$
V_{1}^{d}=\frac{r\left(r+n_{c} \theta_{m p}\right)\left(n_{c} \theta_{m c} u-\gamma_{m}\right)-n_{c}^{2} \theta_{m c} \theta_{m p} \gamma_{m}}{r\left(r+n_{c} \theta_{m p}\right)\left(r+n_{c} \theta_{m c}\right)} .
$$

The deviation is not profitable, and hence $\tau=0$ is an equilibrium, iff $V_{1}^{d} \leq V_{1}$. This reduces to $-\gamma_{m} \geq\left(r+n_{c} \theta_{m p}\right) u$.

Now consider a candidate equilibrium with $\tau=1$. Then we solve in the usual way for

$$
V_{1}=\frac{\left(r+n_{c} \theta_{m p}\right)\left(n_{c} \theta_{m c} u-\gamma_{m}\right)}{r\left(r+n_{c} \theta_{m c}+n_{c} \theta_{m p}\right)} .
$$

A deviation to $\tau=0$ implies $V_{1}^{d}=-\gamma_{m} / r$. This is not profitable, and hence $\tau=1$ is an equilibrium, iff $V_{1}^{d} \leq V_{1}$. This reduces to $-\gamma_{m} \leq\left(r+n_{c} \theta_{m p}\right) u$. Equilibrium is generically unique. 